\title{
Generalized spheroidal wave equation and limiting cases
}

\author{
B. D. Bonorino Figueiredo \\ Instituto de Cosmologia, Relatividade e Astrofísica (ICRA-BR) \\ Centro Brasileiro de Pesquisas Físicas (CBPF) \\ Rua Dr. Xavier Sigaud, 150 - 22290-180 - Rio de Janeiro, RJ, Brasil
}

\begin{abstract}
We find sets of solutions to the generalized spheroidal wave equation (GSWE) or, equivalently, to the confluent Heun equation. Each set is constituted by three solutions, one given by a series of ascending powers of the independent variable, and the others by series of regular and irregular confluent hypergeometric functions. For a fixed set, the solutions converge over different regions of the complex plane but present series coefficients proportional to each other. These solutions for the GSWE afford solutions to a double-confluent Heun equation by a taking-limit process due to Leaver. Another procedure, called Whittaker-Ince limit, provides solutions in series of powers and Bessel functions for two other equations with a different type of singularity at infinity. In addition, new solutions are obtained for the Whittaker-Hill and Mathieu equations by considering these as special cases of both the confluent and doubleconfluent Heun equations. In particular, we find that each of the Lindmann-Stieltjes solutions for the Mathieu equation is associated with two expansions in series of Bessel functions. We also discuss a set of solutions in series of hypergeometric and confluent hypergeometric functions for the GSWE and use their Leaver limits to obtain infinite-series solutions for the Schrödinger equation with an asymmetric double-Morse potential. Finally, the possibility of extending the solutions of the GSWE to the general Heun equation is briefly discussed.
\end{abstract}

\section{Introduction}

We study sets of solutions for a generalized spheroidal wave equation (GSWE) aiming at obtaining solutions for other differential equations which are related to the GSWE by taking-limit processes. Although the three solutions which constitute a fixed set converge over different regions of the complex plane, they have series coefficients proportional to one another what means that, under certain circumstances, the three solutions imply a unique characteristic equation resulting from the three-term recurrence relations for the coefficients. Solutions for a double-confluent Heun equation (DCHE) are generated from the ones for the GSWE by using of a limit process devised by Leaver [1] and, therefore, without solving directly the DCHE. On the other hand, by applying to the GSWE and DCHE another limit due to Whittaker and Ince [2, 3, 4, we obtain two differential equations with a different type of singularity at infinity whose solutions are obtained from the ones for GSWE and DCHE by taking the Whittaker-Ince limit, again without integrating directly the equations. Thus, by means of the Leaver and Whittaker-Ince limits, the GSWE afford solutions for three other different equations.

Furthermore, new solutions for the Whittaker-Hill and Mathieu equations are obtained from the fact that these equations are particular cases of both the GSWE and the DCHE [5]. From one side, this gives two types of solutions for the Whittaker-Hill equation (WHE) and, from the other side, three types for the Mathieu equation in virtue that this one is also an instance of the Whittaker-Ince limit of the GSWE.

In summary, solutions for the GSWE generate solutions for five other equations which in most of the cases have been studied separately and without any connection with the GSWE, namely: DCHE, Ince's limits of the GSWE and DCHE, and the Mathieu and Whittaker-Hill equations. 
Some solutions we shall find are already known, but here they are associated with two other solutions possessing different mathematical properties, since we are dealing with sets containing three solutions, as mentioned in the first paragraph (the known solutions result from a known solution for the GSWE given by a series of ascending powers of the independent variable).

In addition to the solutions for the GSWE and its limiting and particular cases, we consider two physical problems. One is given by the Schrödinger equation for asymmetric double-Morse potentials concerning quantum spin systems [6, 7]. For quasi-exactly solvable potentials [8, 9], an earlier attempt to solve this equation had been unsuccessful [10]. Here we show that the solution of this problem requires the use of two different types of solutions for the DCHE, both of them discussed in this article. The other problem is given by the Teukolsky equations for gravitational backgrounds of black holes, which afford a connection among three equations: general Heun equation, GSWE and DCDE [11, 12]. However, this problem is regarded only at the extent it helps to find solutions for the Heun equation and its confluent cases.

After these general considerations, let us examine each of these six equations and how they are connected, discuss some features of the solutions and outline the structure of the paper.

The generalized spheroidal wave equation (GSWE). For the GSWE we adopt the form used by Leaver [1],

$$
z\left(z-z_{0}\right) \frac{d^{2} U}{d z^{2}}+\left(B_{1}+B_{2} z\right) \frac{d U}{d z}+\left[B_{3}-2 \eta \omega\left(z-z_{0}\right)+\omega^{2} z\left(z-z_{0}\right)\right] U=0,(\omega \neq 0)
$$

where $B_{i}, \eta$ and $\omega$ are constants and $z=0$ and $z=z_{0}$ are regular singular points with indicial exponents $\left(0,1+B_{1} / z_{0}\right)$ and $\left(0,1-B_{2}-B_{1} / z_{0}\right)$, respectively, that is,

$$
\begin{array}{lll}
\lim _{z \rightarrow 0} U(z) \sim 1 & \text { or } \quad \lim _{z \rightarrow 0} U(z) \sim z^{1+\left(B_{1} / z_{0}\right)} \\
\lim _{z \rightarrow z_{0}} U(z) \sim 1 & \text { or } \quad \lim _{z \rightarrow z_{0}} U(z) \sim\left(z-z_{0}\right)^{1-B_{2}-\left(B_{1} / z_{0}\right)} .
\end{array}
$$

At $z=\infty$, which is an irregular singularity, the behavior inferred from the normal Thomé solutions [1, 13] is

$$
\lim _{z \rightarrow \infty} U(z) \sim e^{ \pm i \omega z} z^{\mp i \eta-\left(B_{2} / 2\right)}
$$

Eq. (1) is in a form appropriate for the study of the Teukolsky equations for the Kerr metric since, in the upper limit for the rotation parameter, $z_{0}$ vanishes and the equation reduces to an DCHE. In fact, Eq. (11) is equivalent to the confluent Heun equation [5, 14, 15] and, when $\eta=0$, it reduces to the so-called ordinary spheroidal wave equation [16].

The double-confluent Heun equation (DCHE). By setting $z_{0}=0$ in Eq. (1), Leaver obtained a DCHE with five parameters [1], namely,

$$
z^{2} \frac{d^{2} U}{d z^{2}}+\left(B_{1}+B_{2} z\right) \frac{d U}{d z}+\left(B_{3}-2 \eta \omega z+\omega^{2} z^{2}\right) U=0,\left(B_{1} \neq 0, \omega \neq 0\right),
$$

where $z=0$ and $z=\infty$ are both irregular singularities. At $z=\infty$ the behavior of the solutions is again given by Eq. (3), while at $z=0$ the normal Thomé solutions affords

$$
\lim _{z \rightarrow 0} U(z) \sim 1, \text { or } \lim _{z \rightarrow 0} U(z) \sim e^{B_{1} / z} z^{2-B_{2}} .
$$

The values $B_{1}=0$ and/or $\omega=0$ (for $\eta$ fixed) are excluded because: if $B_{1}=\omega=0$ we have the Euler equation; if $B_{1}=0$ and $\omega \neq 0$ or if $B_{1} \neq 0$ and $\omega=0$, the equation degenerates into a confluent hypergeometric equation (Appendix A of [4]). 
Here the Leaver limit $z_{0} \rightarrow 0$ is used to obtain solutions for the DCHE (41) from solutions of the GSWE. Furthermore, the Leaver form (4) for the DCHE has also the advantage of admitting the Whittaker-Ince limit, in opposition to other forms which have only four parameters, as in Refs. [5, 14, 17].

The Whittaker-Ince limit of the GSWE. We define the Whittaker-Ince limit of the GSWE and DCHE as

$$
\omega \rightarrow 0, \quad \eta \rightarrow \infty, \text { such that } 2 \eta \omega=-q, \quad \text { (Whittaker-Ince limit) }
$$

where $q$ is a constant. In a previous article [4] we have referred to the above as 'Ince limit', but according to Humbert [2, prior to Ince 3], Whittaker had used such procedure to get the Mathieu equation from the Whittaker-Hill one. In any case, the limit (6] $)$ is a generalization of the limit used by Whittaker and Ince.

The Whittaker-Ince limit of the GSWE is

$$
z\left(z-z_{0}\right) \frac{d^{2} U}{d z^{2}}+\left(B_{1}+B_{2} z\right) \frac{d U}{d z}+\left[B_{3}+q\left(z-z_{0}\right)\right] U=0,(q \neq 0)
$$

(if $q=0$ this equation can be transformed into a hypergeometric equation). At the regular singular points $z=0$ and $z=z_{0}$ the behaviors of the solutions are formally the same as those for the GSWE, but at the irregular singular point $z=\infty$, from the subnormal Thomé solutions [13] we get

$$
\lim _{z \rightarrow \infty} U(z) \sim e^{ \pm 2 i \sqrt{q z}} z^{(1 / 4)-\left(B_{2} / 2\right)}
$$

in contrast with the behavior (3) of original GSWE (normal Thomé solutions).

Mignemi [18] and Malmendier [19] have found a particular case $\left(B_{2}=2 B_{1}, \quad z_{0}=-1\right)$ of Eq. (7) for a wave equation resulting from the Laplace-Beltrami operator for a scalar field on the Eguchi-Hanson space [20], but they did not succeed in deriving solutions from solutions of the GSWE. Here and in Ref. [4] this goal is attained by starting with solutions for the GSWE which admit both the Leaver and the Whittaker-Ince limits. One can check that this is not possible for some other solutions as the Hylleraas and Jaffé ones [1, for example.

The Whittaker-Ince limit of the DCHE. The Whittaker-Ince limit of the DCHE is the equation

$$
z^{2} \frac{d^{2} U}{d z^{2}}+\left(B_{1}+B_{2} z\right) \frac{d U}{d z}+\left(B_{3}+q z\right) U=0,\left(q \neq 0, B_{1} \neq 0\right)
$$

where $z=0$ and $z=\infty$ are irregular singularities as in the DCHE (44). At the irregular singular point $z=0$ the solutions behave again according to Eq. (15), but at infinity they admit the behavior given by the subnormal Thomé solutions, Eq. (8) .

Eq. (9) is not obtainable from a DCHE with four parameters. In fact, the scale transformation in the variable $z$ used to pass from five into four parameters [5, 14] becomes meaningless when $\omega=0$ in Eq. (4). So, the Leaver form (41) is valuable since it leads to solutions for Eq. (9) which rules, for example, the radial dependence of the Schrödinger equation for the scattering of low-energy ions by polarizable neutral atoms with an induced quadrupole momentum [4].

In Eq. (9) $q=0$ and/or $B_{1}=0$ were excluded because in these cases the equation can be transformed into a confluent hypergeometric equation if $q=0$ and $B_{1} \neq 0$, into a Bessel equation if $q \neq 0$ and $B_{1}=0$, and into an Euler equation if $q=B_{1}=0$ [4] .

The previous equations - (11), (4), (7) and (9) - can also be distinguished by the rank or species of the singularity at $z=\infty$ [5, 14]. For the GSWE (1) and the DCHE (41) the rank is 1 (species 
2), while for the Whittaker-Ince limits (7) and (9) the rank is $1 / 2$ (species 1 ). However, here this classification is not relevant. To get the solutions, more important are the concepts of Leaver and Whittaker-Ince limits, and the information on whether the solutions exhibit the behavior predicted by either the normal or the subnormal Thomé solutions.

Notice that Eq. (9) is also the Leaver limit of Eq. (7) and, consequently, we have two ways to generate its solutions, as indicated in the following scheme:

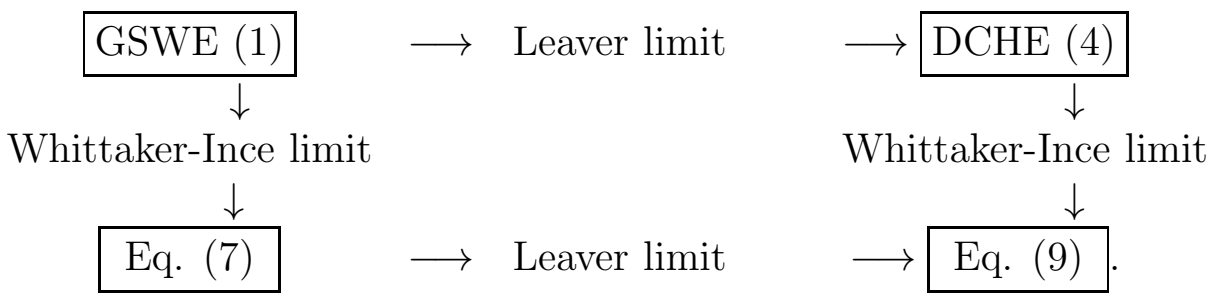

A preliminary study of solutions for the Heun equation indicates that above diagram can be improved by adding the connection Heun equation $\rightarrow$ GSWE (see Appendix B).

Whittaker-Hill and Mathieu equations. The Whittaker-Hill and Mathieu equations are particular cases of both the GSWE (11) and the DCHE (4), but the Mathieu equation is also a special instance of the Whittaker-Ince limit of the GSWE. Thence, there are two types of solutions for the Whittaker-Hill equation (WHE) and three types for the Mathieu equation, as stated above. This result is due to Decarreau, Maroni and Robert [5] but it is necessary to study the properties of the solutions obtained by each of these approaches, by writing such solutions explicitly and comparing them.

To avoid confusion in the notation, we write the Whittaker-Hill and Mathieu equations in a form independent of the parameters $B_{i}, \eta$ or $\omega$ which appear in the other equations. We choose the trigonometric (hyperbolic) form because this is useful to investigate the parity and periodicity properties of such solutions. For the WHE we employ the form [3, 21]

$$
\frac{d^{2} W}{d u^{2}}+\kappa^{2}\left[\vartheta-\frac{1}{8} \xi^{2}-(p+1) \xi \cos (2 \kappa u)+\frac{1}{8} \xi^{2} \cos (4 \kappa u)\right] W=0, \quad(\mathrm{WHE})
$$

where we have written $\vartheta$, instead of the usual $\eta$, because $\eta$ has already appeared in the GSWE and DCHE. If $u$ is a real variable, this equation represents the WHE when $\kappa=1$ and the modified WHE when $\kappa=i$. The WHE (11) is converted into a GSWE or DCHE in Secs. II B or III B, respectively.

For the Mathieu equation we use the form [22]

$$
\frac{d^{2} W}{d u^{2}}+\sigma^{2}\left[a-2 k^{2} \cos (2 \sigma u)\right] W=0, \quad \text { (Mathieu equation) }
$$

where $\sigma=1$ or $\sigma=i$ for the Mathieu or modified Mathieu equation, respectively. Note that there are two ways to get Eq. (12) from the WHE (11). First, for $p=-1$ and $\kappa=\sigma / 2$, the WHE (11) reduces to the Mathieu equation (12). Second, the Mathieu equation (12) also follows from the WHE (11) by means of the original Whittaker-Ince limit [2, 3, 23]: $\xi \rightarrow 0, p \rightarrow \infty$ so that $p \xi=2 k^{2}, \kappa=\sigma$ and $\vartheta=a$. This second way is equivalent to consider the Mathieu equation as a particular case of the Whittaker-Ince limit (7) of the GSWE. The Mathieu equation (12) is transformed into a GSWE, a DCHE or the Ince limit of the GSWE in Secs. II C, III C or IV B, respectively.

In this paper, starting from sets formed by three solutions for the GSWE, we follow the scheme (10) to generate solutions for the other equations. In each set, one solution is given by a BarberHassé expansion in power series [1, 24] while the others are given by expansions in series of regular 
and irregular hypergeometric functions. In a fixed set, the solutions converge over different regions but are collected together because their series coefficients are proportional to each other. The construction of sets of solutions with these features is in part suggested by study of the Mathieu equations [22, but it follows as well from the study of wave equations in general relativity where sometimes is necessary to match solutions convergent over different domains [1, 10, 25, 26, 27, 28.

In the context of the GSWE, a initial set, as the one given in Eq. (33a), leads to the others by means of transformation rules coming from variable substitutions which preserve the form of the GSWE but modify their parameters and/or arguments. The transformation rules for the GSWE, written in Eqs. (13a), allow us to generate solutions with all the expected behaviors at the singular points. These features are transferred to the solutions of the limiting equations and their particular cases. This is illustrated by the results of Sections II B and IV B, where we find that each set of solutions for the Whittaker-Hill and Mathieu equations, respectively, possesses different properties of parity and /or periodicity.

On the other hand, the convergence of the series depends on whether the parameters of the differential equation do or do not satisfy a relationship, known as characteristic equation, which results from the three-term recurrence relations for the series coefficients. In fact, there are two cases to be considered. First, if there is some arbitrary constant in the differential equation, the characteristic equation can be satisfied by using it to determine that constant in terms of the others and in such case the series converges. If there is no disposable constant, the characteristic equation in general cannot be satisfied and then the series diverges. In this second case, the convergence is assured by inserting into certain solutions a parameter $\nu$, called phase or characteristic parameter, which plays the role of the arbitrary constant and must be adjusted so as to validate the characteristic equation. Excepting section VI, all the other sections of the present article deal with solutions without characteristic parameter and, consequently, assume the existence of an arbitrary constant in the differential equations.

In section II A, we study the Barber-Hassé solutions and the expansions in series of confluent hypergeometric functions for the GSWE. In section II B these solutions are particularized for the Whittaker-Hill equation and in Sec. II C for the Mathieu equation. In each set of solutions for the WHE, the solution resulting from the Barber-Hassé one turns out to be a solution already found by Arscott [21], but the others seem to be new.

In Sec. III A the Leaver limit $z_{0} \rightarrow 0$ is used to derive solutions for the DCHE from solutions for the GSWE. The solutions ensuing from the Barber-Hassé solutions include as particular cases the ones which have been investigated by Decareau, Maroni and Robert [14] and also by Schmidt and Wolf [17] for a DCHE with four parameters, but to each of these now correspond two expansions in series of confluent hypergeometric functions. These solutions are used to find finite-series solutions for the Schrödinger equation with an asymmetric double-Morse potential. There are infinite-series solutions but these are not regular at the singular points of the equation. The WHE and the Mathieu equations as particular cases of the DCHE are discussed in Secs. III B and III $\mathrm{C}$, respectively.

In Sec. IV we deal with the Whittaker-Ince limit of the GSWE and find that the expansions in series of confluent hypergeometric functions reduce to expansions in series of Bessel functions. In the special case of the Mathieu equation, we recover some solutions found by Lindmann and Stieltjes [29, 30], each one coupled with two expansions in series of Bessel functions. Similarly, in Sec. $\mathrm{V}$ we establish the Ince limits for the solutions of the DCHE.

In Sec. VI we consider some solutions with a phase parameter for the case in which there is no free constant in the GSWE. Each set of solutions for the GSWE contains two expansions in series of hypergeometric functions and two in series of confluent hypergeometric functions. These solutions also admit of both the Leaver and Whittaker-Ince limits. The solutions for the DCHE 
are used to find regular infinite-series solutions for the Schrödinger equation with the asymmetric double-Morse potential mentioned above. For this it is necessary to match two solutions converging over different ranges of the independent variable but having a common region of convergence. The results is that, for this quasi-exactly potential, the entire spectrum of energies may be determined by solving a characteristic equation given by the sum of two infinite continued fractions.

Section VII contains some concluding remarks, while Appendix A presents an alternative derivation of the expansions in series of Bessel functions for the Whittaker-Ince limit of the GSWE and Appendix B discusses some connections between solutions of the general Heun equation and generalized spheroidal equation.

\section{Solutions to the generalized spheroidal wave equation}

In this section, first we write down transformation rules for the GSWE and recall some basic properties of the three-term recurrence relations for the series coefficients (not restricted to the GSWE). After this we set the solutions for the general case and discuss both the Whittaker-Hill and Mathieu equations from the viewpoint of the GSWE.

In the first place, if $U(z)=U\left(B_{1}, B_{2}, B_{3} ; z_{0}, \omega, \eta ; z\right)$ denotes one solution of the GSWE, the transformation rules $T_{1}, T_{2}, T_{3}$ and $T_{4}$ are given by [4, 31]

$$
\begin{aligned}
& T_{1} U(z)=z^{1+B_{1} / z_{0}} U\left(C_{1}, C_{2}, C_{3} ; z_{0}, \omega, \eta ; z\right), \quad z_{0} \neq 0, \\
& T_{2} U(z)=\left(z-z_{0}\right)^{1-B_{2}-B_{1} / z_{0}} U\left(B_{1}, D_{2}, D_{3} ; z_{0}, \omega, \eta ; z\right), \quad z_{0} \neq 0, \\
& T_{3} U(z)=U\left(B_{1}, B_{2}, B_{3} ; z_{0},-\omega,-\eta ; z\right), \quad \forall z_{0}, \\
& T_{4} U(z)=U\left(-B_{1}-B_{2} z_{0}, B_{2}, B_{3}+2 \eta \omega z_{0} ; z_{0},-\omega, \eta ; z_{0}-z\right), \quad \forall z_{0}
\end{aligned}
$$

where

$$
\begin{aligned}
& C_{1}=-B_{1}-2 z_{0}, \quad C_{2}=2+B_{2}+\frac{2 B_{1}}{z_{0}}, C_{3}=B_{3}+\left(1+\frac{B_{1}}{z_{0}}\right)\left(B_{2}+\frac{B_{1}}{z_{0}}\right), \\
& D_{2}=2-B_{2}-\frac{2 B_{1}}{z_{0}}, D_{3}=B_{3}+\frac{B_{1}}{z_{0}}\left(\frac{B_{1}}{z_{0}}+B_{2}-1\right) .
\end{aligned}
$$

These rules can be verified by substitutions of variables. In $T_{3}$ it is assumed that we must change the sign of $(\eta, \omega)$ only where these quantities appear explicitly in the solution $U(z)$, keeping the expressions for the other parameters unchanged even if they depend on $\eta$ and $\omega$ as in the Teukolsky equations of the relativistic astrophysics [1, 32]. For brevity, we use only $T_{1}$ and $T_{2}$. The rule $T_{4}$ interchanges the position of the regular singular points $\left(z=z_{0} \leftrightarrow z=0\right)$ and may be used to get solutions with the appropriate behavior at $z=0$.

On the other hand, if $b_{n}$ denotes the series coefficients of a solution, then the three-term recurrence relations (for solutions without phase parameter) have the form

$$
\alpha_{0} b_{1}+\beta_{0} b_{0}=0, \quad \alpha_{n} b_{n+1}+\beta_{n} b_{n}+\gamma_{n} b_{n-1}=0(n \geq 1)
$$

where $\alpha_{n}, \beta_{n}$ and $\gamma_{n}$ are constants depending on the parameters of the differential equation. These recurrence relations constitute a infinite system of homogeneous linear equations which can 
be written as

$$
\left(\begin{array}{cccccccc}
\beta_{0} & \alpha_{0} & 0 & \cdots & & & 0 & \cdots \\
\gamma_{1} & \beta_{1} & \alpha_{1} & 0 & & & \vdots & \\
0 & \gamma_{2} & \beta_{2} & \alpha_{2} & & & & \\
\vdots & & & & & & & \\
& & & & \gamma_{n} & \beta_{n} & \alpha_{n} & \cdots \\
& & & & & & \vdots &
\end{array}\right)\left(\begin{array}{l}
b_{0} \\
b_{1} \\
b_{2} \\
\vdots \\
b_{n} \\
\vdots
\end{array}\right)=0
$$

Then, to have nontrivial solutions for $b_{n}$, the (Hill) determinant of the above infinite tridiagonal matrix must vanish. This is possible only if there is some arbitrary parameter in the differential equation, that is, in the elements of the matrix. Equivalently, the recurrence relations lead to characteristic equation in terms of the infinite continued fraction [1]

$$
\beta_{0}=\frac{\alpha_{0} \gamma_{1}}{\beta_{1}-} \frac{\alpha_{1} \gamma_{2}}{\beta_{2}-} \frac{\alpha_{2} \gamma_{3}}{\beta_{3}-} \cdots
$$

So, instead of a vanishing determinant, we must satisfy the characteristic equation and this also assures the series convergence by means of some version of a Perron theorem [33].

Under certain conditions, three-term recurrence relations like (14) give finite-series solutions which are also called quasi-polynomial solutions, Heun polynomials or quasi-algebraic solutions. The condition sufficient for having terminating series is $\gamma_{n}=0$ for some $n=N=$ positive integer [34], in which case the series presents $N$ terms, that is, $0 \leq n \leq N-1$. In effect, if $\gamma_{N}=0$ we can choose the parameters of the equation so that $b_{N}=0$ : then, the relations (14) imply that $b_{n}=0$ for any $n \geq N$. In such event, in general there are infinite series as well since the transformation rules generate solutions with other recurrence relations. In addition, if $\alpha_{n}=0$ for some $n=N$, the series begins at $n=N+1$, but in this case one may take $n=m+N+1$ and relabel the series coefficients in order to obtain a series beginning at $m=0$.

There is another remark which will be useful in Secs. II A and IV A. Suppose a second solution with coefficients $c_{n}$ having the recurrence relations

$$
\tilde{\alpha}_{0} c_{1}+\beta_{0} c_{0}=0, \quad \tilde{\alpha}_{n} c_{n+1}+\beta_{n} c_{n}+\tilde{\gamma}_{n} c_{n-1}=0(n \geq 1)
$$

where $\beta_{n}$ is the same as in Eq. (14). Then, if

$$
\tilde{\alpha}_{n} \tilde{\gamma}_{n+1}=\alpha_{n} \gamma_{n+1}
$$

it is obvious from Eq. (16) that, for infinite series, both solutions have the same characteristic equation provided that the sums begin at $n=0$ in both series.

\section{A. GSWE and the Barber-Hassé solutions}

Now we construct the initial set containing the solutions $\left(U_{1}^{0}, U_{1}^{\infty}, U_{1}\right) . U_{1}^{0}$ is the Barber-Hassé expansion in series of ascending power of $z-z_{0}$ which converges for any finite $z$ and was originally proposed to solve an angular GSWE in which $0 \leq z \leq z_{0}[1]$. $U_{1}^{\infty}$ is given by an expansion in series of irregular confluent hypergeometric functions $\Psi(a, b ; y)$ (or confluent hypergeometric function of second kind), converges for $|z|>\left|z_{0}\right|$ and, thus, is suitable to solve a radial GSWE $\left(z \geq z_{0}\right)$ except at $z=z_{0} . U_{1}$ is an expansion in series of regular confluent hypergeometric functions $\Phi(a, b ; y)$ (or confluent hypergeometric function of first kind) which converges for any value of $z$ but, in general, 
its behavior at infinity is not the one predicted by the Thomé solutions. From the first set of solutions we obtain three other sets by using the transformation rules $T_{1}$ and $T_{2}$.

To obtain the Barber-Hassé solution $U_{1}^{0}$ we write $U(z)=U_{1}^{0}(z)=e^{i \omega z} f(z)$. Then, the GSWE (11) leads to

$$
z\left(z-z_{0}\right) \frac{d^{2} f}{d z^{2}}+\left[B_{1}+B_{2} z+2 i \omega z\left(z-z_{0}\right)\right] \frac{d f}{d z}+\left[B_{3}+i \omega B_{1}+i \omega B_{2} z-2 \omega \eta\left(z-z_{0}\right)\right] f=0 .
$$

Now, by expanding $f(z)$ in a Frobenius series corresponding to the exponent 0 about $z=z_{0}$, i. e.,

$$
f(z)=\sum_{n=0}^{\infty} b_{n}^{(1)}\left(z-z_{0}\right)^{n} \Rightarrow U_{1}^{0}(z)=e^{i \omega z} \sum_{n=0}^{\infty} b_{n}^{(1)}\left(z-z_{0}\right)^{n},
$$

we find that $b_{n}^{(1)}$ satisfy the recurrence relations $\left(b_{-1}^{(1)}=0\right)$

$$
\begin{aligned}
z_{0}\left(n+B_{2}+\frac{B_{1}}{z_{0}}\right)(n+1) & b_{n+1}^{(1)}+\left[n\left(n+B_{2}-1+2 i \omega z_{0}\right)+i \omega z_{0}\left(B_{2}+\frac{B_{1}}{z_{0}}\right)+B_{3}\right] b_{n}^{(1)} \\
+ & 2 i \omega\left(n-1+i \eta+\frac{B_{2}}{2}\right) b_{n-1}^{(1)}=0 .
\end{aligned}
$$

This solution can also be obtained by applying the rule $T_{4}$ to the Barber-Hassé expansion given in Ref. [1]. It converges for any finite $z$ provided that the characteristic equation is fulfilled [1, 24].

To find the solutions in series of confluent hypergeometric functions, we carry out the substitutions

$$
U(z)=e^{i \omega z} F(y), \quad y=-2 i \omega z
$$

which transform the GSWE (1) into

$$
\begin{array}{r}
\left(y+2 i \omega z_{0}\right)\left[y \frac{d^{2} F}{d y^{2}}-y \frac{d F}{d y}\right]+B_{2} y \frac{d F}{d y}-2 i \omega B_{1} \frac{d F}{d y}+ \\
{\left[B_{3}+i \omega B_{1}+2 \eta \omega z_{0}-\left(i \eta+\frac{B_{2}}{2}\right) y\right] F=0 .}
\end{array}
$$

Then, first we expand $F(y)$ in series of the irregular functions $\Psi(a, b ; y)$ [35] according to

$$
\begin{aligned}
& F(y)=\sum_{n=0}^{\infty} c_{n}^{(1)} \Psi\left(n+i \eta+\frac{B_{2}}{2}, n+B_{2} ; y\right)=\sum_{n=0}^{\infty} c_{n}^{(1)} \Psi_{n}(y) \Rightarrow \\
& U_{1}^{\infty}(z)=e^{i \omega z} \sum_{n=0}^{\infty} c_{n}^{(1)} \Psi\left(n+i \eta+\frac{B_{2}}{2}, n+B_{2} ;-2 i \omega z\right),
\end{aligned}
$$

where $\Psi_{n}(y)=\Psi\left(n+i \eta+B_{2} / 2, n+B_{2} ; y\right)$. These $\Psi_{n}(y)$ satisfy

$$
\begin{aligned}
& y \frac{d^{2} \Psi_{n}(y)}{d y^{2}}-y \frac{d \Psi_{n}(y)}{d y}=-\left(n+B_{2}\right) \frac{d \Psi_{n}(y)}{d y}+\left(n+i \eta+\frac{B_{2}}{2}\right) \Psi_{n}(y), \\
& \frac{d \Psi_{n}(y)}{d y}=-\left(n+i \eta+\frac{B_{2}}{2}\right) \Psi_{n+1}(y), \\
& y \frac{d \Psi_{n}(y)}{d y}=\left(1-n-B_{2}+y\right) \Psi_{n}(y)-\Psi_{n-1}(y), \\
& \left(n+i \eta+\frac{B_{2}}{2}\right) y \Psi_{n+1}(y)+\left(1-n-B_{2}+y\right) \Psi_{n}(y)-\Psi_{n-1}(y)=0,
\end{aligned}
$$


where the first relation is the confluent hypergeometric equation for $\Psi_{n}(y)$ and the others follow from the properties of the irregular confluent hypergeometric functions [35]. Thus, inserting the previous expression for $F(y)$ into Eq. (21b) and using Eqs. (23), we obtain

$$
\sum_{n=1}^{\infty} n c_{n}^{(1)} \Psi_{n-1}(y)+\sum_{n=0}^{\infty} c_{n}^{(1)}\left[\beta_{n}^{(1)} \Psi_{n}(y)+2 i \omega z_{0}\left(n+B_{2}+\frac{B_{1}}{z_{0}}\right)\left(n+i \eta+\frac{B_{2}}{2}\right) \Psi_{n+1}(y)\right]=0
$$

where $\beta_{n}^{(1)}$ is the coefficient of $b_{n}^{(1)}$ in the recurrence relation for the solution $U_{1}^{0}$, namely,

$$
\beta_{n}^{(1)}=n\left(n+B_{2}-1+2 i \omega z_{0}\right)+i \omega z_{0}\left(B_{2}+\frac{B_{1}}{z_{0}}\right)+B_{3}
$$

Putting $m=n-1$ and $m=n+1$ in the first and last terms of Eq. (24), respectively, we find

$$
\begin{gathered}
{\left[c_{1}^{(1)}+\beta_{0}^{(1)} c_{0}^{(1)}\right] \Psi_{0}(y)+} \\
\sum_{m=1}^{\infty}\left[(m+1) c_{m+1}^{(1)}+\beta_{m}^{(1)} c_{m}^{(1)}+2 i \omega z_{0}\left(m-1+B_{2}+\frac{B_{1}}{z_{0}}\right)\left(m-1+i \eta+\frac{B_{2}}{2}\right) c_{m-1}^{(1)}\right] \Psi_{m}(y)=0 .
\end{gathered}
$$

By equating to zero the coefficients of each independent $\Psi_{n}(y)$ we find the three-term recurrence relations $\left(c_{-1}^{(1)}=0\right)$

$$
(n+1) c_{n+1}^{(1)}+\beta_{n}^{(1)} c_{n}^{(1)}+2 i \omega z_{0}\left(n-1+B_{2}+\frac{B_{1}}{z_{0}}\right)\left(n-1+i \eta+\frac{B_{2}}{2}\right) c_{n-1}^{(1)}=0, \quad n \geq 0 .
$$

From the behavior of $\Psi(a, b ; y)$, when $b \rightarrow \infty$ while $b-a$ and $y$ remain bounded [35], we get

$$
\lim _{n \rightarrow \infty} \frac{\Psi_{n+1}(y)}{\Psi_{n}(y)}=\frac{1}{2 i \omega z} .
$$

Besides this, using a Perron-Kreuser theorem [33] we find that the minimal solution of the recurrence relations (25) satisfies

$$
\lim _{n \rightarrow \infty} \frac{c_{n+1}^{(1)}}{c_{n}^{(1)}}= \begin{cases}-2 i \omega z_{0}, & \text { if } z_{0} \neq 0 \\ -2 i \omega B_{1} / n, & \text { if } z_{0}=0\end{cases}
$$

Then, combining these two limits, we have

$$
\lim _{n \rightarrow \infty} \frac{c_{n+1}^{(1)} \Psi_{n+1}(y)}{c_{n}^{(1)} \Psi_{n}(y)}= \begin{cases}-z_{0} / z, & \text { if } z_{0} \neq 0 \\ -B_{1} /(n z), & \text { if } z_{0}=0\end{cases}
$$

Therefore, by the ratio test, the series in $U_{1}^{\infty}(z)$ converges for any $|z|>\left|z_{0}\right|$ on the condition that the characteristic equation is satisfied. The behavior of $U_{i}^{\infty}(z)$ when $z \rightarrow \infty$ results from the relation 35 ]

$$
\lim _{y \rightarrow \infty} \Psi(a, b ; y) \sim y^{-a}\left[1+O\left(|y|^{-1}\right)\right], \quad-\frac{3 \pi}{2}<\arg y<-\frac{3 \pi}{2}
$$

which implies

$$
\lim _{z \rightarrow \infty} U_{1}^{\infty}(z) \sim e^{i \omega z} z^{-i \eta-\left(B_{2} / 2\right)}, \quad-\frac{3 \pi}{2}<\arg (-2 i \omega z)<\frac{3 \pi}{2}
$$


as in a normal Thomé solution.

Now we expand $F(y)$ in series of regular confluent hypergeometric functions $\Phi(a, b ; y)$. If we define the function $\hat{\Phi}_{n}(y)$ as

$$
\hat{\Phi}_{n}(y)=\frac{(-1)^{n}}{\Gamma\left(n+B_{2}\right)} \Phi\left(n+i \eta+\frac{B_{2}}{2}, n+B_{2} ; y\right), y=-2 i \omega z
$$

and use some difference and differential properties of $\Phi(a, b ; y)$ [36], we find that $\hat{\Phi}_{n}(z)$ also satisfy the relations (23). Therefore, substituting $\hat{\Phi}_{n}(y)$ for $\Psi_{n}(y)$ in Eq. (22) we get the solution

$$
U_{1}(z)=e^{i \omega z} \sum_{n=0}^{\infty} c_{n}^{(1)} \hat{\Phi}_{n}(y)=e^{i \omega z} \sum_{n=0}^{\infty} c_{n}^{(1)} \hat{\Phi}\left(n+i \eta+\frac{B_{2}}{2}, n+B_{2} ;-2 i \omega z\right)
$$

where the coefficients $c_{n}^{(1)}$ are the same which appear in the solution $U_{1}^{\infty}(z)$. In order to show that the series in $U_{1}(z)$ converges for any $z$, we use the behavior of $\Phi(a, b ; y)$ when $b \rightarrow \infty$ while $b-a$ and $y$ remain bounded [35, namely,

$$
\lim _{b \rightarrow \infty} \Phi(a, b ; y)=e^{y}\left[1+O\left(|b|^{-1}\right)\right], \quad(b-a=\text { finite })
$$

which leads to

$$
\lim _{n \rightarrow \infty} \frac{\hat{\Phi}_{n+1}(y)}{\hat{\Phi}_{n}(y)}=-\frac{1}{n+B_{2}}
$$

Thus, using again the minimal solution of the recurrence relations for $c_{n}^{(1)}$ we get

$$
\lim _{n \rightarrow \infty} \frac{c_{n+1}^{(1)} \hat{\Phi}_{n+1}(y)}{c_{n}^{(1)} \hat{\Phi}_{n}(y)}= \begin{cases}\frac{2 i \omega z_{0}}{n+B_{2}} \rightarrow 0, & \text { if } z_{0} \neq 0 \\ \frac{2 i \omega B_{1}}{n\left(n+B_{2}\right)} \rightarrow 0, & \text { if } z_{0}=0\end{cases}
$$

Therefore, the ratio test implies that the series in the solution $U_{1}(z)$ converges for any value of $z$. However, the behavior of $U_{1}(z)$ when $z \rightarrow \infty$ in general does not coincide with the one inferred from the Thomé solutions, since we have [36]

$$
\lim _{y \rightarrow \infty} \Phi(a, b ; y)= \begin{cases}\frac{\Gamma(b)}{\Gamma(a)} e^{y} y^{a-b}\left[1+O\left(|y|^{-1}\right)\right], & (\Re y>0) \\ \frac{\Gamma(b)}{\Gamma(b-a)}(-y)^{-a}\left[1+O\left(|y|^{-1}\right)\right], & (\Re y<0) .\end{cases}
$$

Thus, only if $\Re y<0$ the asymptotic behavior of $U_{1}$ has the same form as $U_{1}^{\infty}$. If $\Re y=0$, the limit of $\Phi(a, b ; y)$ is a combination of the two expressions given on the right-hand side of the previous expression [35]. On the other hand, although the function $\Phi(a, b ; y)$ is not defined if $b=0,-1,-2, \cdots$, the function $\hat{\Phi}(a, b ; y)$ is defined since [35]

$$
\lim _{b \rightarrow 1-m} \frac{\Phi(a, b ; y)}{\Gamma(b)}=\frac{(a)_{m}}{m !} y^{m} \Phi(a+m, 1+m ; y), \quad m=1,2,3, \cdots,
$$

$(a)_{m}=a(a+1) \cdots(a+m-1)$ for $m \geq 1$. Therefore, the expansion $U_{1}(z)$ is defined even if $B_{2}$ is zero or a negative integer.

In the following we collect the three solutions in the first set of solutions, and this gives three other sets by the use of the transformation rules $T_{1}$ and $T_{2}$ according to

$$
\left(U_{1}^{0}, U_{1}^{\infty}, U_{1}\right) \stackrel{T_{1}}{\longleftrightarrow}\left(U_{2}^{0}, U_{2}^{\infty}, U_{2}\right) \stackrel{T_{2}}{\longleftrightarrow}\left(U_{3}^{0}, U_{3}^{\infty}, U_{3}\right) \stackrel{T_{1}}{\longleftrightarrow}\left(U_{4}^{0}, U_{4}^{\infty}, U_{4}\right) \stackrel{T_{2}}{\longleftrightarrow}\left(U_{1}^{0}, U_{1}^{\infty}, U_{1}\right) .
$$


First set. This set admits both the Leaver and Ince limits. If $B_{2}+B_{1} / z_{0}=1$, it is equal to the fourth set.

$$
\begin{aligned}
& U_{1}^{0}(z)=e^{i \omega z} \sum_{n=0}^{\infty} b_{n}^{(1)}\left(z-z_{0}\right)^{n}, \\
& U_{1}^{\infty}(z)=e^{i \omega z} \sum_{n=0}^{\infty} c_{n}^{(1)} \Psi\left(n+i \eta+\frac{B_{2}}{2}, n+B_{2} ;-2 i \omega z\right), \\
& U_{1}(z)=e^{i \omega z} \sum_{n=0}^{\infty} c_{n}^{(1)} \hat{\Phi}\left(n+i \eta+\frac{B_{2}}{2}, n+B_{2} ;-2 i \omega z\right) .
\end{aligned}
$$

Setting $b_{-1}^{(1)}=0$ and $c_{-1}^{(1)}=0$, the three-term recurrence relations for the coefficients are given by

$$
\begin{gathered}
z_{0}\left(n+B_{2}+\frac{B_{1}}{z_{0}}\right)(n+1) b_{n+1}^{(1)}+\left[n\left(n+B_{2}-1+2 i \omega z_{0}\right)+i \omega z_{0}\left(B_{2}+\frac{B_{1}}{z_{0}}\right)+B_{3}\right] b_{n}^{(1)} \\
+2 i \omega\left(n-1+i \eta+\frac{B_{2}}{2}\right) b_{n-1}^{(1)}=0
\end{gathered}
$$

and

$$
\begin{aligned}
& (n+1) c_{n+1}^{(1)}+\left[n\left(n+B_{2}-1+2 i \omega z_{0}\right)+i \omega z_{0}\left(B_{2}+\frac{B_{1}}{z_{0}}\right)+B_{3}\right] c_{n}^{(1)}+ \\
& 2 i \omega z_{0}\left(n-1+B_{2}+\frac{B_{1}}{z_{0}}\right)\left(n-1+i \eta+\frac{B_{2}}{2}\right) c_{n-1}^{(1)}=0 .
\end{aligned}
$$

The coefficients $b_{n}^{(1)}$ and $c_{n}^{(1)}$ are connected by

$$
c_{n}^{(1)}=\left(z_{0}\right)^{n} \Gamma\left(n+B_{2}+\frac{B_{1}}{z_{0}}\right) b_{n}^{(1)}, \text { if } B_{2}+\frac{B_{1}}{z_{0}} \neq 0,-1,-2, \cdots
$$

apart from a multiplicative constant independent of $n$. The restrictions on $B_{2}+\left(B_{1} / z_{0}\right)$ assure that the sum in the three solutions begins at $n=0$ and, thus, they have the same characteristic equation for the reason given in the paragraph containing Eqs. (17a) and (17b). In addition, we have finite-series solutions if $i \eta+\left(B_{2} / 2\right)$ is zero or negative integer.

Second set. These solutions admit the Ince limit but not the Leaver limit. If $B_{2}+B_{1} / z_{0}=1$, the second and the third sets are equal to one another.

$$
\begin{aligned}
& U_{2}^{0}(z)=e^{i \omega z} z^{1+\frac{B_{1}}{z_{0}}} \sum_{n=0}^{\infty} b_{n}^{(2)}\left(z-z_{0}\right)^{n}, \\
& U_{2}^{\infty}(z)=e^{i \omega z} z^{1+\frac{B_{1}}{z_{0}}} \sum_{n=0}^{\infty} c_{n}^{(2)} \Psi\left(n+1+i \eta+\frac{B_{1}}{z_{0}}+\frac{B_{2}}{2}, n+2+B_{2}+\frac{2 B_{1}}{z_{0}} ;-2 i \omega z\right), \\
& U_{2}(z)=e^{i \omega z} z^{1+\frac{B_{1}}{z_{0}}} \sum_{n=0}^{\infty} c_{n}^{(2)} \hat{\Phi}\left(n+1+i \eta+\frac{B_{1}}{z_{0}}+\frac{B_{2}}{2}, n+2+B_{2}+\frac{2 B_{1}}{z_{0}} ;-2 i \omega z\right),
\end{aligned}
$$

where the recurrence relations are

$$
\begin{gathered}
z_{0}\left(n+B_{2}+\frac{B_{1}}{z_{0}}\right)(n+1) b_{n+1}^{(2)}+ \\
{\left[n\left(n+1+2 i \omega z_{0}+B_{2}+\frac{2 B_{1}}{z_{0}}\right)+i \omega z_{0}\left(B_{2}+\frac{B_{1}}{z_{0}}\right)+\left(1+\frac{B_{1}}{z_{0}}\right)\left(B_{2}+\frac{B_{1}}{z_{0}}\right)+B_{3}\right] b_{n}^{(2)}} \\
+2 i \omega\left(n+i \eta+\frac{B_{1}}{z_{0}}+\frac{B_{2}}{2}\right) b_{n-1}^{(2)}=0,
\end{gathered}
$$


and

$$
\begin{gathered}
(n+1) c_{n+1}^{(2)}+ \\
{\left[n\left(n+1+2 i \omega z_{0}+B_{2}+\frac{2 B_{1}}{z_{0}}\right)+i \omega z_{0}\left(B_{2}+\frac{B_{1}}{z_{0}}\right)+\left(1+\frac{B_{1}}{z_{0}}\right)\left(B_{2}+\frac{B_{1}}{z_{0}}\right)+B_{3}\right] c_{n}^{(2)}} \\
+2 i \omega\left(n-1+B_{2}+\frac{B_{1}}{z_{0}}\right)\left(n+i \eta+\frac{B_{1}}{z_{0}}+\frac{B_{2}}{2}\right) c_{n-1}^{(2)}=0 .
\end{gathered}
$$

The coefficients are connected by

$$
c_{n}^{(2)}=\left(z_{0}\right)^{n} \Gamma\left(n+B_{2}+\frac{B_{1}}{z_{0}}\right) b_{n}^{(2)}, \text { if } B_{2}+\frac{B_{1}}{z_{0}} \neq-0,-1,-2, \cdots .
$$

Third set. These solutions admit both the Leaver and Ince limits.

$$
\begin{aligned}
& U_{3}^{0}(z)=e^{i \omega z} z^{1+\frac{B_{1}}{z_{0}}}\left(z-z_{0}\right)^{1-B_{2}-\frac{B_{1}}{z_{0}}} \sum_{n=0}^{\infty} b_{n}^{(3)}\left(z-z_{0}\right)^{n} \\
& U_{3}^{\infty}(z)=e^{i \omega z} z^{1+\frac{B_{1}}{z_{0}}}\left(z-z_{0}\right)^{1-B_{2}-\frac{B_{1}}{z_{0}}} \sum_{n=0}^{\infty} c_{n}^{(3)} \Psi\left(n+2+i \eta-\frac{B_{2}}{2}, n+4-B_{2} ;-2 i \omega z\right) \\
& U_{3}(z)=e^{i \omega z} z^{1+\frac{B_{1}}{z_{0}}}\left(z-z_{0}\right)^{1-B_{2}-\frac{B_{1}}{z_{0}}} \sum_{n=0}^{\infty} c_{n}^{(3)} \hat{\Phi}\left(n+2+i \eta-\frac{B_{2}}{2}, n+4-B_{2} ;-2 i \omega z\right)
\end{aligned}
$$

where the recurrence relations are given by

$$
\begin{gathered}
z_{0}\left(n+2-B_{2}-\frac{B_{1}}{z_{0}}\right)(n+1) b_{n+1}^{(3)} \\
+\left[n\left(n+3+2 i \omega z_{0}-B_{2}\right)+i \omega z_{0}\left(2-B_{2}-\frac{B_{1}}{z_{0}}\right)+2-B_{2}+B_{3}\right] b_{n}^{(3)} \\
+2 i \omega\left(n+1+i \eta-\frac{B_{2}}{2}\right) b_{n-1}^{(3)}=0
\end{gathered}
$$

and

$$
\begin{gathered}
(n+1) c_{n+1}^{(3)}+\left[n\left(n+3+2 i \omega z_{0}-B_{2}\right)+i \omega z_{0}\left(2-B_{2}-\frac{B_{1}}{z_{0}}\right)+2-B_{2}+B_{3}\right] c_{n}^{(3)}+ \\
2 i \omega\left(n+1-B_{2}-\frac{B_{1}}{z_{0}}\right)\left(n+1+i \eta-\frac{B_{2}}{2}\right) c_{n-1}^{(3)}=0 .
\end{gathered}
$$

In this case, we find

$$
c_{n}^{(3)}=\left(z_{0}\right)^{n} \Gamma\left(n+2-B_{2}-\frac{B_{1}}{z_{0}}\right) b_{n}^{(3)}, \text { if } B_{2}+\frac{B_{1}}{z_{0}} \neq 2,3,4, \cdots .
$$

Fourth set. This set admits the Ince limit but not the Leaver limit.

$$
\begin{aligned}
& U_{4}^{0}(z)=e^{i \omega z}\left(z-z_{0}\right)^{1-B_{2}-\frac{B_{1}}{z_{0}}} \sum_{n=0}^{\infty} b_{n}^{(4)}\left(z-z_{0}\right)^{n} \\
& U_{4}^{\infty}(z)=e^{i \omega z}\left(z-z_{0}\right)^{1-B_{2}-\frac{B_{1}}{z_{0}}} \sum_{n=0}^{\infty} c_{n}^{(4)} \Psi\left(n+1+i \eta-\frac{B_{1}}{z_{0}}-\frac{B_{2}}{2}, n+2-B_{2}-\frac{2 B_{1}}{z_{0}} ;-2 i \omega z\right), \\
& U_{4}(z)=e^{i \omega z}\left(z-z_{0}\right)^{1-B_{2}-\frac{B_{1}}{z_{0}}} \sum_{n=0}^{\infty} c_{n}^{(4)} \hat{\Phi}\left(n+1+i \eta-\frac{B_{1}}{z_{0}}-\frac{B_{2}}{2}, n+2-B_{2}-\frac{2 B_{1}}{z_{0}} ;-2 i \omega z\right),
\end{aligned}
$$


with the recurrence relations

$$
\begin{gathered}
z_{0}\left(n+2-B_{2}-\frac{B_{1}}{z_{0}}\right)(n+1) b_{n+1}^{(4)}+ \\
{\left[n\left(n+1+2 i \omega z_{0}-B_{2}-\frac{2 B_{1}}{z_{0}}\right)+i \omega z_{0}\left(2-B_{2}-\frac{B_{1}}{z_{0}}\right)+\frac{B_{1}}{z_{0}}\left(B_{2}+\frac{B_{1}}{z_{0}}-1\right)+B_{3}\right]} \\
\times b_{n}^{(4)}+2 i \omega\left(n+i \eta-\frac{B_{1}}{z_{0}}-\frac{B_{2}}{2}\right) b_{n-1}^{(4)}=0
\end{gathered}
$$

and

$$
\begin{gathered}
(n+1) c_{n+1}^{(4)}+ \\
{\left[n\left(n+1+2 i \omega z_{0}-B_{2}-\frac{2 B_{1}}{z_{0}}\right)+i \omega z_{0}\left(2-B_{2}-\frac{B_{1}}{z_{0}}\right)+\frac{B_{1}}{z_{0}}\left(B_{2}+\frac{B_{1}}{z_{0}}-1\right)+B_{3}\right] c_{n}^{(4)}+} \\
2 i \omega z_{0}\left(n+1-B_{2}-\frac{B_{1}}{z_{0}}\right)\left(n+i \eta-\frac{B_{1}}{z_{0}}-\frac{B_{2}}{2}\right) c_{n-1}^{(4)}=0 .
\end{gathered}
$$

This time the connecting formula is

$$
c_{n}^{(4)}=\left(z_{0}\right)^{n} \Gamma\left(n+2-B_{2}-\frac{B_{1}}{z_{0}}\right) b_{n}^{(4)}, \text { if } B_{2}+\frac{B_{1}}{z_{0}} \neq 2,3,4, \cdot .
$$

As the rule $T_{4}$ changes the position of the regular singular points, it may be used to get solutions with the suitable behavior at $z=0$. For these new sets of solutions, the $U_{i}^{0}(i=5,6,7,8)$ again converge for any finite value of $z$, the $U_{i}^{\infty}$ converge for $\left|z-z_{0}\right|>\left|z_{0}\right|$, whereas the $U_{i}$ converge for all values of $z$.

The previous convergence properties are transferred to the limiting and particular cases of the GSWE. The utility of the convergence of the solutions $U_{i}$ over the entire complex plane requires further investigation. In fact, other solutions converging for any $z$ (also giving by series of regular confluent hypergeometric functions) have already been found in Sec. VII of Ref. [1]. In any case, in physical problems we have also to consider boundary and regularity conditions, in addition to convergence.

\section{B. Whittaker-Hill equation as a GSWE and Arscott's solutions}

By inserting $z=\cos ^{2}(\kappa u)$ and $W(u)=U(z)$ into the WHE (11), we obtain the GSWE

$$
z(z-1) \frac{d^{2} U}{d z^{2}}+\left(-\frac{1}{2}+z\right) \frac{d U}{d z}+\left[\frac{1}{4}(p+1) \xi-\frac{\vartheta}{4}+\frac{(p+1) \xi}{2}(z-1)-\frac{\xi^{2}}{4} z(z-1)\right] U=0 .
$$

From this and Eq. (11), we find that the solutions $W(u)$ for the WHE (11) may be written in terms of the solutions $U(z)$ of the GSWE (1) as follows

$$
\left.\begin{array}{l}
W(u)=U(z), \quad z=\cos ^{2}(\kappa u)(\kappa=1, i) ; \\
z_{0}=1, B_{1}=-\frac{1}{2}, B_{2}=1, B_{3}=\frac{1}{4}[(p+1) \xi-\vartheta], i \omega=\frac{\xi}{2}, i \eta=\frac{p+1}{2}
\end{array}\right\} \begin{aligned}
& \text { WHE as } \\
& \text { GSWE. }
\end{aligned}
$$

To obtain the solutions for the WHE in terms of $z$ it is sufficient to replace the parameters given above into the solutions $U(z)$ of the GSWE, but to get the periodicity and parity properties of the solutions we also use the transformation $z=\cos ^{2}(\kappa u)$. 
First set. Even solutions, period $\pi$ if $\kappa=1$ and $u=$ real (if $\kappa=1 W_{1}^{\infty}$ does not converge).

$$
\begin{array}{ll}
W_{1}^{0}(u)=e^{\frac{\xi}{2} \cos ^{2}(\kappa u)} \sum_{n=0}^{\infty} \frac{(-1)^{n}}{\Gamma[n+(1 / 2)]} c_{n}^{(1)} \sin ^{2 n}(\kappa u), & |\cos (\kappa u)|<\infty, \\
W_{1}^{\infty}(u)=e^{\frac{\xi}{2} \cos ^{2}(\kappa u)} \sum_{n=0}^{\infty} c_{n}^{(1)} \Psi\left(n+1+\frac{p}{2}, n+1 ;-\xi \cos ^{2}(\kappa u)\right), & |\cos (\kappa u)|>1, \\
W_{1}(u)=e^{\frac{\xi}{2} \cos ^{2}(\kappa u)} \sum_{n=0}^{\infty} c_{n}^{(1)} \hat{\Phi}\left(n+1+\frac{p}{2}, n+1 ;-\xi \cos ^{2}(\kappa u)\right), & \forall u
\end{array}
$$

where the recurrence relations for $c_{n}^{(1)}$ are

$$
(n+1) c_{n+1}^{(1)}+\left[n(n+\xi)+\frac{2 \xi+p \xi-\vartheta}{4}\right] c_{n}^{(1)}+\xi\left(n-\frac{1}{2}\right)\left(n+\frac{p}{2}\right) c_{n-1}^{(1)}=0 .
$$

Second set. Even solutions with period $2 \pi$ if $\kappa=1$.

$$
\begin{array}{ll}
W_{2}^{0}(u)=\cos (\kappa u) e^{\frac{\xi}{2} \cos ^{2}(\kappa u)} \sum_{n=0}^{\infty} \frac{(-1)^{n}}{\Gamma[n+(1 / 2)]} c_{n}^{(2)} \sin ^{2 n}(\kappa u), & |\cos (\kappa u)|<\infty, \\
W_{2}^{\infty}(u)=\cos (\kappa u) e^{\frac{\xi}{2} \cos ^{2}(\kappa u)} \sum_{n=0}^{\infty} c_{n}^{(2)} \Psi\left(n+\frac{3}{2}+\frac{p}{2}, n+2 ;-\xi \cos ^{2}(\kappa u)\right), & |\cos (\kappa u)|>1, \\
W_{2}(u)=\cos (\kappa u) e^{\frac{\xi}{2} \cos ^{2}(\kappa u)} \sum_{n=0}^{\infty} c_{n}^{(2)} \hat{\Phi}\left(n+\frac{3}{2}+\frac{p}{2}, n+2 ;-\xi \cos ^{2}(\kappa u)\right), & \forall u
\end{array}
$$

with the recurrence relations

$$
(n+1) c_{n}^{(2)}+\left[n(n+1+\xi)+\frac{1+2 \xi+p \xi-\vartheta}{4}\right] c_{n}^{(2)}+\xi\left(n-\frac{1}{2}\right)\left(n+\frac{p}{2}+\frac{1}{2}\right) c_{n-1}^{(2)}=0 .
$$

Third set. Odd solutions, period $\pi$ if $\kappa=1$.

$$
\begin{array}{ll}
W_{3}^{0}(u)=\sin (2 \kappa u) e^{\frac{\xi}{2} \cos ^{2}(\kappa u)} \sum_{n=0}^{\infty} \frac{(-1)^{n}}{\Gamma[n+(3 / 2)]} c_{n}^{(3)} \sin ^{2 n}(\kappa u), & |\cos (\kappa u)|<\infty, \\
W_{3}^{\infty}(u)=\sin (2 \kappa u) e^{\frac{\xi}{2} \cos ^{2}(\kappa u)} \sum_{n=0}^{\infty} c_{n}^{(3)} \Psi\left(n+2+\frac{p}{2}, n+3 ;-\xi \cos ^{2}(\kappa u)\right), & |\cos (\kappa u)|>1, \\
W_{3}(u)=\sin (2 \kappa u) e^{\frac{\xi}{2} \cos ^{2}(\kappa u)} \sum_{n=0}^{\infty} c_{n}^{(3)} \hat{\Phi}\left(n+2+\frac{p}{2}, n+3 ;-\xi \cos ^{2}(\kappa u)\right), & \forall u
\end{array}
$$

having the recurrence relations

$$
(n+1) c_{n+1}^{(3)}+\left[n(n+2+\xi)+1+\xi+\frac{p \xi-\vartheta}{4}\right] c_{n}^{(3)}+\xi\left(n+\frac{1}{2}\right)\left(n+1+\frac{p}{2}\right) c_{n-1}^{(3)}=0
$$


Fourth set. Odd solutions with period $2 \pi$ if $\kappa=1$.

$$
\begin{array}{ll}
W_{4}^{0}(u)=\sin (\kappa u) e^{\frac{\xi}{2} \cos ^{2}(\kappa u)} \sum_{n=0}^{\infty} \frac{(-1)^{n}}{\Gamma[n+(3 / 2)]} c_{n}^{(4)} \sin ^{2 n}(\kappa u), & |\cos (\kappa u)|<\infty, \\
W_{4}^{\infty}(u)=\sin (\kappa u) e^{\frac{\xi}{2} \cos ^{2}(\kappa u)} \sum_{n=0}^{\infty} c_{n}^{(4)} \Psi\left(n+\frac{3}{2}+\frac{p}{2}, n+2 ;-\xi \cos ^{2}(\kappa u)\right), & |\cos (\kappa u)|>1, \\
W_{4}(u)=\sin (\kappa u) e^{\frac{\xi}{2} \cos ^{2}(\kappa u)} \sum_{n=0}^{\infty} c_{n}^{(4)} \hat{\Phi}\left(n+\frac{3}{2}+\frac{p}{2}, n+2 ;-\xi \cos ^{2}(\kappa u)\right), & \forall u
\end{array}
$$

where the recurrence relations are

$$
(n+1) c_{n+1}^{(4)}+\left[n(n+1+\xi)+\xi+\frac{1+p \xi-\vartheta}{4}\right] c_{n}^{(4)}+\xi\left(n+\frac{1}{2}\right)\left(n+\frac{1}{2}+\frac{p}{2}\right) c_{n-1}^{(4)}=0 .
$$

The solutions corresponding to the opposite choice for the signs of $\omega$ and $\eta$ in Eqs. (42) are obtained by the substitutions

$$
\xi \rightarrow-\xi, p \rightarrow-p-2,
$$

which take the place of the rule $T_{3}$. The operation equivalent to the rule $T_{4}$ is

$$
u \rightarrow u+\frac{\pi}{2 \kappa}, \quad \xi \rightarrow-\xi
$$

Similarly, the composition $T_{3} T_{4}$ corresponds to the symmetry

$$
u \rightarrow u+\frac{\pi}{2 \kappa}, \quad p \rightarrow-p-2,
$$

of the WHE. In fact, if we apply this last operation to the preceding sets of solutions, we find the solutions $W_{i}^{0}$ obtained by Arscott [21], each one accompanied by two expansions in series of hypergeometric functions.

Notice that the use of the transformations $T_{1}$ and $T_{2}$ have been essential to generate a subgroup containing four sets of solutions which are even or odd and have period $\pi$ or $2 \pi$. The solutions for WHE obtained from the one for DCHE do not possess these properties of parity and periodicity (see Sec. III B).

\section{Mathieu equation as a GSWE}

Although the Whittaker-Ince limit of the preceding solutions for Whittaker-Hill equation gives solutions for the Mathieu equation, such solutions are also particular cases of the solutions for the Whittaker-Ince limit of the GSWE and, for this reason, will be discussed in Sec. IV B. Here we regard the solutions $W(u)$ for the Mathieu equation (12) as a particular case (instead of a limiting case) of the solutions for the GSWE. Such solutions $W(u)$ may be obtained by

$$
\left.\begin{array}{l}
W(u)=U(z), z=\cos ^{2}\left(\frac{\sigma u}{2}\right), \quad(\sigma=1, i), \\
z_{0}=1, B_{1}=-\frac{1}{2}, B_{2}=1, B_{3}=2 k^{2}-a, \omega= \pm 4 k, \quad \eta=0
\end{array}\right\} \begin{aligned}
& \text { Mathieu equation } \\
& \text { as GSWE, }
\end{aligned}
$$

where $U(z)$ are the solutions for GSWE given in Sec. II A, or, equivalently, by writing

$$
\kappa=\sigma / 2, p=-1, \vartheta=4 a-8 k^{2}, \xi= \pm 8 i k \quad \text { (Mathieu equation as WHE) }
$$


into the solutions $W(u)$ for the WHE, given in Sec. II B. These solutions are also even or odd but their period are twice the period of the solutions corresponding to the WHE (if $\sigma=1$ ), that is, one has period $2 \pi$ and $4 \pi$. As usual, there is no finite-series solutions.

First set. Even solutions with period $2 \pi$ if $\sigma=1$.

$$
\begin{array}{ll}
W_{1}^{0}(u)=e^{ \pm 4 i k \cos ^{2}(\sigma u / 2)} \sum_{n=0}^{\infty} \frac{(-1)^{n}}{\Gamma[n+(1 / 2)]} c_{n}^{(1)} \sin ^{2 n} \frac{\sigma u}{2}, & \left|\cos \frac{\sigma u}{2}\right|<\infty, \\
W_{1}^{\infty}(u)=e^{ \pm 4 i k \cos ^{2}(\sigma u / 2)} \sum_{n=0}^{\infty} c_{n}^{(1)} \Psi\left(n+\frac{1}{2}, n+1 ; \mp 8 i k \cos ^{2} \frac{\sigma u}{2}\right), & \left|\cos \frac{\sigma u}{2}\right|>1, \\
W_{1}(u)=e^{ \pm 4 i k \cos ^{2}(\sigma u / 2)} \sum_{n=0}^{\infty} c_{n}^{(1)} \hat{\Phi}\left(n+\frac{1}{2}, n+1 ; \mp 8 i k \cos ^{2} \frac{\sigma u}{2}\right), & \forall u
\end{array}
$$

where the recurrence relations for the coefficients are

$$
(n+1) c_{n+1}^{(1)}+\left[n(n \pm 8 i k) \pm 2 i k+2 k^{2}-a\right] c_{n}^{(1)} \pm 8 i k\left(n-\frac{1}{2}\right)^{2} c_{n-1}^{(1)}=0
$$

In these and in the following solutions the upper or the lower sign must be taken throughout.

Second set. Even solutions, period $4 \pi$ if $\sigma=1$

$$
\begin{array}{ll}
W_{2}^{0}(u)=\cos (\sigma u / 2) e^{ \pm 4 i k \cos ^{2}(\sigma u / 2)} \sum_{n=0}^{\infty} \frac{(-1)^{n}}{\Gamma[n+(1 / 2)]} c_{n}^{(2)} \sin ^{2 n} \frac{\sigma u}{2}, & \left|\cos \frac{\sigma u}{2}\right|<\infty, \\
W_{2}^{\infty}(u)=\cos (\sigma u / 2) e^{ \pm 4 i k \cos ^{2}(\sigma u / 2)} \sum_{n=0}^{\infty} c_{n}^{(2)}\left(\mp 8 i k \cos ^{2} \frac{\sigma u}{2}\right)^{-n-1}, & \left|\cos \frac{\sigma u}{2}\right|>1, \\
W_{2}(u)=\cos (\sigma u / 2) e^{ \pm 4 i k \cos ^{2}(\sigma u / 2)} \sum_{n=0}^{\infty} c_{n}^{(2)} \hat{\Phi}\left(n+1, n+2 ; \mp 8 i k \cos ^{2} \frac{\sigma u}{2}\right), & \forall u
\end{array}
$$

with the recurrence relations

$$
(n+1) c_{n}^{(2)}+\left[n(n+1 \pm 8 i k)+\frac{1}{4} \pm 2 i k+2 k^{2}-a\right] c_{n}^{(2)} \pm 8 i k n\left(n-\frac{1}{2}\right) c_{n-1}^{(2)}=0
$$

Note that in $W_{2}^{\infty}(u)$ we have eliminated the function $\Psi\left(n+1, n+2 ; \mp 8 i k \cos ^{2}(\sigma u / 2)\right)$ by using the integral representation [36]

$$
\Gamma(a) \Psi(a, b ; x)=\int_{0}^{\infty} e^{-x t} t^{a-1}(1+t)^{b-a-1} d t, \quad(\Re b>\Re a>0)
$$

along with the definite integral [37]

$$
\int_{0}^{\infty} t^{n} e^{-\alpha t} d t=\frac{\Gamma(n+1)}{\alpha^{n+1}}
$$


Third set. Odd solutions, period $2 \pi$ if $\sigma=1$.

$$
\begin{array}{ll}
W_{3}^{0}(u)=\sin (\sigma u) e^{ \pm 4 i k \cos ^{2}(\sigma u / 2)} \sum_{n=0}^{\infty} \frac{(-1)^{n}}{\Gamma[n+(3 / 2)]} c_{n}^{(3)} \sin ^{2 n} \frac{\sigma u}{2}, & \left|\cos \frac{\sigma u}{2}\right|<\infty, \\
W_{3}^{\infty}(u)=\sin (\sigma u) e^{ \pm 4 i k \cos ^{2}(\sigma u / 2)} \sum_{n=0}^{\infty} c_{n}^{(3)} \Psi\left(n+\frac{3}{2}, n+3 ; \mp 8 i k \cos ^{2} \frac{\sigma u}{2}\right), & \left|\cos \frac{\sigma u}{2}\right|>1, \\
W_{3}(u)=\sin (\sigma u) e^{ \pm 4 i k \cos ^{2}(\sigma u / 2)} \sum_{n=0}^{\infty} c_{n}^{(3)} \hat{\Phi}\left(n+\frac{3}{2}, n+3 ; \mp 8 i k \cos ^{2} \frac{\sigma u}{2}\right), & \forall u
\end{array}
$$

having the recurrence relations

$$
(n+1) c_{n+1}^{(3)}+\left[n(n+2 \pm 8 i k)+1 \pm 6 i k+2 k^{2}-a\right] c_{n}^{(3)} \pm 8 i k\left(n+\frac{1}{2}\right)^{2} c_{n-1}^{(3)}=0 .
$$

Fourth set. Odd solutions, period $4 \pi$ if $\sigma=1$.

$$
\begin{array}{ll}
W_{4}^{0}(u)=\sin (\sigma u / 2) e^{ \pm 4 i k \cos ^{2}(\sigma u / 2)} \sum_{n=0}^{\infty} \frac{(-1)^{n}}{\Gamma[n+(3 / 2)]} c_{n}^{(4)} \sin ^{2 n} \frac{\sigma u}{2}, & \left|\cos \frac{\sigma u}{2}\right|<\infty, \\
W_{4}^{\infty}(u)=\sin (\sigma u / 2) e^{ \pm 4 i k \cos ^{2}(\sigma u / 2)} \sum_{n=0}^{\infty} c_{n}^{(4)}\left(\mp 8 i k \cos ^{2} \frac{\sigma u}{2}\right)^{-n-1}, & \left|\cos \frac{\sigma u}{2}\right|>1, \\
W_{4}(u)=\sin (\sigma u / 2) e^{ \pm 4 i k \cos ^{2}(\sigma u / 2)} \sum_{n=0}^{\infty} c_{n}^{(4)} \hat{\Phi}\left(n+\frac{3}{2}, n+2 ; \mp 8 i k \cos ^{2} \frac{\sigma u}{2}\right), & \forall u
\end{array}
$$

with

$$
(n+1) c_{n+1}^{(4)}+\left[n(n+1 \pm 8 i k)+\frac{1}{4} \pm 6 i k+2 k^{2}-a\right] c_{n}^{(4)} \pm 8 i k n\left(n+\frac{1}{2}\right) c_{n-1}^{(4)}=0 .
$$

Finally, further solutions are obtained by performing the substitution

$$
u \rightarrow u+\frac{\pi}{\sigma}
$$

into the above solutions. This substitution, which leaves the Mathieu equation (12) invariant, plays the role of the transformation $T_{3} T_{4}$, for it transforms $z=\cos ^{2}(\sigma u / 2)$ into $z=\sin ^{2}(\sigma u / 2)$ without changing $\omega$.

\section{Solutions for the double-confluent Heun equation}

In the present section, first we obtain two sets of solutions for the DCHE

$$
z^{2} \frac{d^{2} U}{d z^{2}}+\left(B_{1}+B_{2} z\right) \frac{d U}{d z}+\left(B_{3}-2 \eta \omega z+\omega^{2} z^{2}\right) U=0,\left(B_{1} \neq 0, \omega \neq 0\right),
$$

by taking the Leaver limit $z_{0} \rightarrow 0$ of the first and third sets solutions for the GSWE given in Sec II A. After examining the general case and discussing finite-series solutions for a Schrödinger equations with an asymmetric double-Morse potential, we establish the properties of these solutions for the Whittaker-Hill and Mathieu equations. 
The following $U_{1}^{0}$ and $U_{2}^{0}$ are, in fact, the normal Thomé solutions in the neighborhood of $z=0$. As mentioned before, they encompass solutions which were investigated by Decareau, Maroni and Robert [14] and also by Schmidt and Wolf [17] for a DCHE with four parameters. However, each of these solutions, resulting of the Baber-Hassé expansions, are now associated with two expansions in series of confluent hypergeometric functions and these converge in the vicinity of $z=\infty$.

\section{A. General case and solutions of Decarreau et al.}

When $z_{0} \rightarrow 0$, the limits of the solutions $U_{1}^{\infty}$ and $U_{1}$ given in Eq. (33a) are obtained immediately. The limit of $U_{1}^{0}$ yields the expression

$$
U_{1}^{0}(z)=e^{i \omega z} \sum_{n=0}^{\infty} b_{n}^{(1)} z^{n}
$$

where the recurrence relations for $b_{n}^{(1)}$ are

$$
B_{1}(n+1) b_{n+1}^{(1)}+\left[n\left(n+B_{2}-1\right)+i \omega B_{1}+B_{3}\right] b_{n}^{(1)}+2 i \omega\left(n+i \eta+\frac{B_{2}}{2}-1\right) b_{n-1}^{(1)}=0 .
$$

Then, by writing $b_{n}^{(1)}=\left(B_{1}\right)^{-n} c_{n}^{(1)}$, we find the solutions given in Eqs. (57a) and (57b).

First set.

$$
\begin{aligned}
& U_{1}^{0}(z)=e^{i \omega z} \sum_{n=0}^{\infty} c_{n}^{(1)}\left(\frac{z}{B_{1}}\right)^{n}, \\
& U_{1}^{\infty}(z)=e^{i \omega z} \sum_{n=0}^{\infty} c_{n}^{(1)} \Psi\left(n+i \eta+\frac{B_{2}}{2}, n+B_{2} ;-2 i \omega z\right), \\
& U_{1}(z)=e^{i \omega z} \sum_{n=0}^{\infty} c_{n}^{(1)} \hat{\Phi}\left(n+i \eta+\frac{B_{2}}{2}, n+B_{2},-2 i \omega z\right),
\end{aligned}
$$

where the recurrence for relations $c_{n}^{(1)}$ are $\left(c_{-1}^{(1)}=0\right)$

$$
(n+1) c_{n+1}^{(1)}+\left[n\left(n+B_{2}-1\right)+i \omega B_{1}+B_{3}\right] c_{n}^{(1)}+2 i \omega B_{1}\left(n+i \eta+\frac{B_{2}}{2}-1\right) c_{n-1}^{(1)}=0 .
$$

Second set. To obtain the Leaver limit of the solutions given in Eqs. (37a) we note that

$$
z^{1+\frac{B_{1}}{z_{0}}}\left(z-z_{0}\right)^{1-B_{2}-\frac{B_{1}}{z_{0}}}=z\left(z-z_{0}\right)^{1-B_{2}}\left(1-\frac{z_{0}}{z}\right)^{-\frac{B_{1}}{z_{0}}} \rightarrow z^{2-B_{2}} e^{B_{1} / z}, \quad\left(z_{0} \rightarrow 0\right) .
$$

Hence, replacing $b_{n}^{(3)}$ by $\left(-B_{1}\right)^{-n} c_{n}^{(3)}$, solutions (37a) lead to

$$
\begin{aligned}
& U_{2}^{0}(z)=e^{i \omega z+\left(B_{1} / z\right)} z^{2-B_{2}} \sum_{n=0}^{\infty} c_{n}^{(2)}\left(-\frac{z}{B_{1}}\right)^{n} \\
& U_{2}^{\infty}(z)=e^{i \omega z+\left(B_{1} / z\right)} z^{2-B_{2}} \sum_{n=0}^{\infty} c_{n}^{(2)} \Psi\left(n+2+i \eta-\frac{B_{2}}{2}, n+4-B_{2},-2 i \omega z\right), \\
& U_{2}(z)=e^{i \omega z+\left(B_{1} / z\right)} z^{2-B_{2}} \sum_{n=0}^{\infty} c_{n}^{(2)} \hat{\Phi}\left(n+2+i \eta-\frac{B_{2}}{2}, n+4-B_{2},-2 i \omega z\right),
\end{aligned}
$$


with the recurrence relations

$$
\begin{aligned}
& (n+1) c_{n+1}^{(2)}+\left[n\left(n+3-B_{2}\right)+2-i \omega B_{1}-B_{2}+B_{3}\right] c_{n}^{(2)} \\
& -2 i \omega B_{1}\left(n+1+i \eta-\frac{B_{2}}{2}\right) c_{n-1}^{(2)}=0 .
\end{aligned}
$$

From the previous solutions we may obtain others by the changes $(\eta, \omega) \leftrightarrow(-\eta,-\omega)$.

Schrödinger equation with asymmetric double-Morse potential. Now we consider the timeindependent Schrödinger equation for particle with mass $m$ and energy $E$,

$$
\frac{d^{2} \psi}{d u^{2}}+[\mathcal{E}-V(u)] \psi=0, \quad u:=\lambda x, \quad \mathcal{E}:=\frac{2 m E}{\hbar^{2} \lambda^{2}}
$$

where $\lambda$ is a real constant, $x$ is the spatial coordinate and $V(u)$ is a function proportional to the potential. For the asymmetric double-Morse potential considered by Zaslavskii and Ulyanov [6, 7 ] $V(u)$ is given by

$$
V(u)=\frac{B^{2}}{4}\left(\sinh u-\frac{C}{B}\right)^{2}-B\left(s+\frac{1}{2}\right) \cosh u
$$

where $B>0, C>0$ and $s$ is a non-negative integer or half-integer. This is said to be a quasiexactly solvable potential because a part of the energy spectrum and corresponding eigenfunctions can be found exactly in closed form [8, 9]. Here this means that one part of the energy spectrum is determined from the recurrence relations of an eigenfunction given by finite series. In section VI B, we show that the other portion can be obtained from the recurrence relations associated with an infinite-series eigenfunction.

For the potential (60) the Schrödinger equation assumes the form

$$
\frac{d^{2} \psi}{d u^{2}}+\left[\mathcal{E}-\frac{C^{2}}{4}+\frac{B^{2}}{8}-\frac{B^{2}}{16} e^{-2 u}-\frac{B}{2}\left(\frac{C}{2}-\frac{1}{2}-s\right) e^{-u}+\frac{B}{2}\left(\frac{C}{2}+\frac{1}{2}+s\right) e^{u}-\frac{B^{2}}{16} e^{2 u}\right] \psi=0
$$

The substitutions [10, 38]

$$
z=e^{u}, \psi(u)=\phi(z)=e^{-B /(4 z)} z^{(C / 2)-s} U(z), z \in[0, \infty)
$$

transform the previous equation into the DCHE (4)

$z^{2} \frac{d^{2} U}{d z^{2}}+\left[\frac{B}{2}+(1+C-2 s) z\right] \frac{d U}{d z}+\left[\mathcal{E}+\frac{B^{2}}{8}+s^{2}-C s+\frac{B}{2}\left(\frac{C}{2}+\frac{1}{2}+s\right) z-\frac{B^{2}}{16} z^{2}\right] U=0$,

and then we can choose the following set of parameters

$$
B_{1}=\frac{B}{2}, B_{2}=1+C-2 s, B_{3}=\mathcal{E}+\frac{B^{2}}{8}+s^{2}-s C, i \omega=-\frac{B}{4}, i \eta=-\frac{C}{2}-\frac{1}{2}-s .
$$

Hence, $\psi(u)=\phi(z)$ is constructed by inserting into Eq. (61) solutions of the DCHE with the parameters specified in Eq. (62) and demanding that these eigenfuctions satisfy the regularity conditions

$$
\lim _{z \rightarrow 0} \phi(z)=0, \lim _{z \rightarrow \infty} \phi(z)=0
$$


Then, from the first set of solutions for the DCHE, we find

$$
\begin{aligned}
& \phi_{1}^{0}(z)=z^{\frac{C}{2}-s} \exp \left[-\frac{B}{4}\left(z+\frac{1}{z}\right)\right] \sum_{n=0}^{\infty} b_{n}^{(1)}\left(\frac{2 z}{B}\right)^{n}, \\
& \phi_{1}^{\infty}(z)=z^{\frac{C}{2}-s} \exp \left[-\frac{B}{4}\left(z+\frac{1}{z}\right)\right] \sum_{n=0}^{\infty} b_{n}^{(1)} \Psi\left(n-2 s, n+1+C-2 s ; \frac{B z}{2}\right),
\end{aligned}
$$

whose recurrence relations for $b_{n}^{(1)}$ are

$$
(n+1) b_{n+1}^{(1)}+\left[n(n+C-2 s)+\mathcal{E}+s^{2}-s C\right] b_{n}^{(1)}-\frac{B^{2}}{4}(n-2 s-1) b_{n-1}^{(1)}=0, \quad b_{-1}^{(1)}=0 .
$$

Since $s$ is a non-negative integer or half-integer, these are finite-series solutions for the reason given at the beginning of Section II. In effect, the coefficient $\gamma_{n}^{(1)}$ of $b_{n-1}^{(1)}$ vanishes for $n=2 s+1$ and consequently the summation is restricted to the range $0 \leq n \leq 2 s$. The regularity conditions are satisfied by both solutions because $B>0$ in the exponential factor; however only one solution is necessary to solve the problem (the series of regular hypergeometric functions is also appropriate). For a given value of $s$, the energies may be found by computing the determinant of the tridiagonal matrix (15) corresponding to Eq. (65), with $0 \leq n \leq 2 s$.

First, notice that the above solutions impose no additional restriction on the parameter $C$, contrary to the solutions employed in earlier approach[10]. Second, the solutions obtained from the other set of solutions for the DCHE are given by infinite-series but do not satisfy the regularity conditions: a suitable two-sided solution $(-\infty<n<\infty)$ is presented in section VI. B. Third, for $C=0$ the potential becomes a symmetric double-Morse potential while the Schrödinger equation reduces to the Whittaker-Hill equation. In this case, by considering the WHE as a GSWE, we find regular infinite-series solutions given by one-sided series, that is, series with $n \geq 0$ [10].

\section{B. Whittaker-Hill equation as a DCHE}

Here we find that the solutions for the Whittaker-Hill, considered as a DCHE, possess no definite parity, contrary to the solutions which result from the GSWE (Sec. II B). Besides this, the periodicity (if any) will depend on the value of the parameter $p$.

The substitutions

$$
z=e^{2 i \kappa u}, \quad W(u)=z^{1+(p / 2)} e^{\xi /(8 z)} U(z)
$$

transform the WHE

$$
\frac{d^{2} W}{d u^{2}}+\kappa^{2}\left[\vartheta-\frac{1}{8} \xi^{2}-(p+1) \xi \cos (2 \kappa u)+\frac{1}{8} \xi^{2} \cos (4 \kappa u)\right] W=0,
$$

into

$$
z^{2} \frac{d^{2} U}{d z^{2}}+\left[-\frac{\xi}{4}+(p+3) z\right] \frac{d U}{d z}+\left[\left(\frac{p}{2}+1\right)^{2}+\frac{\xi^{2}}{32}-\frac{\vartheta}{4}+\frac{(p+1) \xi}{8} z-\frac{\xi^{2}}{64} z^{2}\right] U=0 .
$$

Then, by comparing this with the DCHE (4) we conclude that the solutions $W(u)$ for the WHE are given by

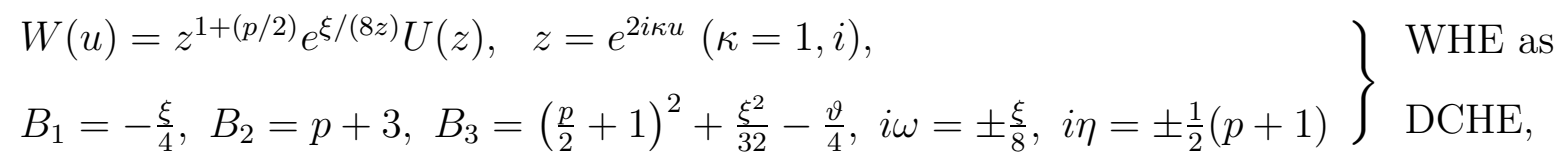


where now $U(z)$ denotes solutions for the DCHE with the parameters specified in the second line of the above expressions.

First set. Taking $i \omega=\xi / 8$ and $i \eta=(p+1) / 2$ and using the solutions (57a), we find

$$
\begin{array}{ll}
W_{1}^{0}(u)=e^{(\xi / 4) \cos (2 \kappa u)+i(2+p) \kappa u} \sum_{n=0}^{\infty}\left(-\frac{4}{\xi}\right)^{n} c_{n}^{(1)} e^{2 i \kappa n u}, & \left|e^{2 i \kappa u}\right|<\infty, \\
W_{1}^{\infty}(u)=e^{(\xi / 4) \cos (2 \kappa u)+i(2+p) \kappa u} \sum_{n=0}^{\infty} \Psi\left(n+p+2, n+p+3 ;-\frac{\xi}{4} e^{2 i \kappa u}\right), & \left|e^{2 i \kappa u}\right|>0, \\
W_{1}(u)=e^{(\xi / 4) \cos (2 \kappa u)+i(2+p) \kappa u} \sum_{n=0}^{\infty} c_{n}^{(1)} \hat{\Phi}\left(n+p+2, n+p+3 ;-\frac{\xi}{4} e^{2 i \kappa u}\right), & \forall u,
\end{array}
$$

where the recurrence relations $c_{n}^{(1)}$ are $\left(c_{-1}^{(1)}=0\right)$

$$
(n+1) c_{n+1}^{(1)}+\left[n(n+p+2)+\left(\frac{p}{2}+1\right)^{2}-\frac{v}{4}\right] c_{n}^{(1)}-\frac{\xi^{2}}{16}(n+p+1) c_{n-1}^{(1)}=0 .
$$

If $p$ is a negative integer equal or less than -2 , the series in these solutions are finite since the coefficient of $c_{n-1}^{(1)}$ vanishes for some value of $n$. For $\kappa=1$ the period of the solutions depends on the factor $\exp (i(p+2) u)$. For instance, the period is: $\pi$ if $p$ is an even integer; $2 \pi$ if $p$ is odd; $2 m \pi(m>1)$ if $p=l / m$, where $l$ and $m$ are prime to one another with $l<m$.

Second set. Taking again $i \omega=\xi / 8$ and $i \eta=(p+1) / 2$ but using the solutions (58a)), we find

$$
\begin{array}{ll}
W_{2}^{0}(u)=e^{(i \xi / 4) \sin (2 \kappa u)-i p \kappa u} \sum_{n=0}^{\infty}\left(\frac{4}{\xi}\right)^{n} c_{n}^{(2)} e^{2 i n \kappa u}, & \left|e^{2 i \kappa u}\right|<\infty, \\
W_{2}^{\infty}(u)=e^{(i \xi / 4) \sin (2 \kappa u)-i p \kappa u} \sum_{n=0}^{\infty} c_{n}^{(2)} \Psi\left(n+1, n+1-p ;-\frac{\xi}{4} e^{2 i \kappa u}\right), & \left|e^{2 i \kappa u}\right|>0, \\
W_{2}(u)=e^{(i \xi / 4) \sin (2 \kappa u)-i p \kappa u} \sum_{n=0}^{\infty} c_{n}^{(2)} \hat{\Phi}\left(n+1, n+1-p ;-\frac{\xi}{4} e^{2 i \kappa u}\right), & \forall u,
\end{array}
$$

where the recurrence relations for $c_{n}^{(2)}$ are

$$
(n+1) c_{n+1}^{(2)}+\left[n(n-p)+\frac{\xi^{2}}{16}+\frac{p^{2}}{4}-\frac{v}{4}\right] c_{n}^{(2)}+\frac{\xi^{2} n}{16} c_{n-1}^{(2)}=0 .
$$

For these solutions the series are infinite.

Third set. Taking $i \omega=-\xi / 8$ and $i \eta=-(p+1) / 2$ and using the solutions (57a), we find

$$
\begin{array}{ll}
W_{3}^{0}(u)=e^{-(i \xi / 4) \sin (2 \kappa u)+i(p+2) \kappa u} \sum_{n=0}^{\infty}\left(-\frac{4}{\xi}\right)^{n} c_{n}^{(3)} e^{2 i n \kappa u}, & \left|e^{2 i \kappa u}\right|<\infty, \\
W_{3}^{\infty}(u)=e^{-(i \xi / 4) \sin (2 \kappa u)+i(p+2) \kappa u} \sum_{n=0}^{\infty} c_{n}^{(3)} \Psi\left(n+1, n+3+p ; \frac{\xi}{4} e^{2 i \kappa u}\right), & \left|e^{2 i \kappa u}\right|>0, \\
W_{3}(u)=e^{-(i \xi / 4) \sin (2 \kappa u)-i p \kappa u} \sum_{n=0}^{\infty} c_{n}^{(3)} \hat{\Phi}\left(n+1, n+3+p ; \frac{\xi}{4} e^{2 i \kappa u}\right), & \forall u,
\end{array}
$$


where the recurrence relations for $c_{n}^{(3)}$ are

$$
(n+1) c_{n+1}^{(3)}+\left[n(n+2+p)+\frac{\xi^{2}}{16}+\left(1+\frac{p}{2}\right)^{2}-\frac{v}{4}\right] c_{n}^{(3)}+\frac{\xi^{2} n}{16} c_{n-1}^{(3)}=0 .
$$

This set can also be obtained from the second one by $p \rightarrow-p-2, \xi \rightarrow-\xi$. For these solutions the series are infinite.

Fourth set. Taking $i \omega=-\xi / 8$ and $i \eta=-(p+1) / 2$ and using the solutions (58a), we get

$$
\begin{array}{ll}
W_{4}^{0}(u)=e^{-(\xi / 4) \cos (2 \kappa u)-i p \kappa u} \sum_{n=0}^{\infty}\left(\frac{4}{\xi}\right)^{n} c_{n}^{(4)} e^{2 i \kappa n u}, & \left|e^{2 i \kappa u}\right|<\infty, \\
W_{4}^{\infty}(u)=e^{-(\xi / 4) \cos (2 \kappa u)-i p \kappa u} \sum_{n=0}^{\infty} c_{n}^{(4)} \Psi\left(n-p, n+1-p ; \frac{\xi}{4} e^{2 i \kappa u}\right), & \left|e^{2 i \kappa u}\right|>0, \\
W_{4}(u)=e^{-(\xi / 4) \cos (2 \kappa u)-i p \kappa u} \sum_{n=0}^{\infty} c_{n}^{(4)} \hat{\Phi}\left(n-p, n+1-p ; \frac{\xi}{4} e^{2 i \kappa u}\right), & \forall u,
\end{array}
$$

where the recurrence relations $c_{n}^{(4)}$ are

$$
(n+1) c_{n+1}^{(4)}+\left[n(n-p)+\frac{p^{2}}{4}-\frac{v}{4}\right] c_{n}^{(4)}-\frac{\xi^{2}}{16}(n-p-1) c_{n-1}^{(4)}=0 .
$$

This set also follows from the first one by the changes $\xi \rightarrow-\xi$ and $p \rightarrow-p-2$. If $p$ is a positive integer, the series in these solutions are finite.

\section{Mathieu equation as a DCHE}

The solutions $W(u)$ for the Mathieu equations (12) are obtained from the solutions given in Sec. III B for the WHE by means of

$$
p=-1,2 \kappa=\sigma, v=4 a-8 k^{2}, \xi= \pm 8 i k .
$$

Unlike the solutions given in Sec. II.3, now there is no solution with definite parity and for $\sigma=1$ ( $u$ real) the period is $4 \pi$ instead of $2 \pi$ and $4 \pi$. We get rid of the functions $\Psi$ which appear in $W_{1}^{\infty}$ and $W_{2}^{\infty}$ by writing

$$
\Psi\left(n+1, n+2 ; \pm 2 i k e^{i \sigma u}\right)=\left( \pm \frac{i}{2 k}\right)^{n+1} e^{-i \sigma(n+1) u}
$$

where we have used equations (52) and (53).

First set. This comes from the first or fourth set of solutions for the WHE.

$$
\begin{array}{ll}
W_{1}^{0}(u)=e^{ \pm 2 i k \cos (\sigma u)+(i / 2) \sigma u} \sum_{n=0}^{\infty}\left( \pm \frac{i}{2 k}\right)^{n} c_{n}^{(1)} e^{i \sigma n u}, & \left|e^{i \sigma u}\right|<\infty, \\
W_{1}^{\infty}(u)=e^{ \pm 2 i k \cos (\sigma u)-(i / 2) \sigma u} \sum_{n=0}^{\infty}\left( \pm \frac{i}{2 k}\right)^{n} c_{n}^{(1)} e^{-i \sigma n u}, & \left|e^{i \sigma u}\right|>0, \\
W_{1}(u)=e^{ \pm 2 i k \cos (\sigma u)+(i / 2) \sigma u} \sum_{n=0}^{\infty} c_{n}^{(1)} \hat{\Phi}\left(n+1, n+2 ; \mp 2 i k e^{i \sigma u}\right), & \forall u,
\end{array}
$$


where the recurrence relations for $c_{n}^{(1)}$ are $\left(c_{-1}^{(1)}=0\right)$

$$
(n+1) c_{n+1}^{(1)}+\left[n(n+1)+\frac{1}{4}+2 k^{2}-a\right] c_{n}^{(1)}+\left(4 k^{2} n\right) c_{n-1}^{(1)}=0 .
$$

Second set. This results from the second or third set of solutions for the WHE.

$$
\begin{array}{ll}
W_{2}^{0}(u)=e^{\mp 2 k \sin (\sigma u)+(i / 2) \sigma u} \sum_{n=0}^{\infty}\left(\mp \frac{i}{2 k}\right)^{n} c_{n}^{(2)} e^{i \sigma n u}, & \left|e^{i \sigma u}\right|<\infty, \\
W_{2}^{\infty}(u)=e^{\mp 2 k \sin (\sigma u)-(i / 2) \sigma u} \sum_{n=0}^{\infty}\left(\mp \frac{i}{2 k}\right)^{n} c_{n}^{(2)} e^{-i \sigma n u}, & \left|e^{i \sigma u}\right|>0, \\
W_{2}(u)=e^{\mp 2 k \sin (\sigma u)+(i / 2) \sigma u} \sum_{n=0}^{\infty} c_{n}^{(2)} \hat{\Phi}\left(n+1, n+2 ; \mp 2 i k e^{i \sigma u}\right), & \forall u,
\end{array}
$$

where the recurrence relations for $c_{n}^{(2)}$ are $\left(c_{-1}^{(2)}=0\right)$

$$
(n+1) c_{n+1}^{(2)}+\left[n(n+1)+\frac{1}{4}-2 k^{2}-a\right] c_{n}^{(2)}-\left(4 k^{2} n\right) c_{n-1}^{(2)}=0 .
$$

\section{Whittaker-Ince's limits for the GSWE}

Four sets of solutions for the Whittaker-Ince limit (7) of the GSWE, that is, for

$$
z\left(z-z_{0}\right) \frac{d^{2} U}{d z^{2}}+\left(B_{1}+B_{2} z\right) \frac{d U}{d z}+\left[B_{3}+q\left(z-z_{0}\right)\right] U=0,(q \neq 0),
$$

result from the solutions given in Sec. II A by means of the very Whittaker-Ince limit $(\omega \rightarrow 0$, $\eta \rightarrow \infty$ such that $2 \eta \omega=-2 q)$. However, we consider only the limit of the first set and, then, use transformation rules to obtain the other sets of solutions. In effect, if $U(z)=U\left(B_{1}, B_{2}, B_{3} ; z_{0}, q ; z\right)$ denotes one solution for Eq. (74), the others are obtained by using the rules

$$
\begin{aligned}
& T_{1} U(z)=z^{1+B_{1} / z_{0}} U\left(C_{1}, C_{2}, C_{3} ; z_{0}, q ; z\right), \quad z_{0} \neq 0, \\
& T_{2} U(z)=\left(z-z_{0}\right)^{1-B_{2}-B_{1} / z_{0}} U\left(B_{1}, D_{2}, D_{3} ; z_{0}, q ; z\right), \quad z_{0} \neq 0, \\
& T_{4} U(z)=U\left(-B_{1}-B_{2} z_{0}, B_{2}, B_{3}-q z_{0} ; z_{0},-q ; z_{0}-z\right),
\end{aligned}
$$

where $C_{i}$ and $D_{i}$ are defined as section II. We again use only $T_{1}$ and $T_{2}$ but notice that $T_{4}$ may be used to get alternative representations for the solutions. As a particular case we set up solutions for the Mathieu equation.

\section{A. General case}

First we rewrite the solutions given in Eq. (33a) as

$$
\begin{aligned}
& U_{1}^{0}(z)=e^{i \omega z} \sum_{n=0}^{\infty} b_{n}^{(1)}\left(z-z_{0}\right)^{n}, \\
& U_{1}^{\infty}(z)=e^{i \omega z} \sum_{n=0}^{\infty} c_{n}^{(1)} \Gamma\left[1+i \eta-\frac{B_{2}}{2}\right] \Psi\left(n+i \eta+\frac{B_{2}}{2}, n+B_{2} ;-\frac{q z}{i \eta}\right), \\
& U_{1}(z)=e^{i \omega z} \sum_{n=0}^{\infty} c_{n}^{(1)} \hat{\Phi}\left(n+i \eta+\frac{B_{2}}{2}, n+B_{2} ;-\frac{q z}{i \eta}\right),
\end{aligned}
$$


where we have multiplied the solution $U_{1}^{\infty}(z)$ by $\Gamma\left[1+i \eta-\frac{B_{2}}{2}\right]$ and written $q=-2 \eta \omega$. From these solutions and respective recurrence relations for their coefficients, the limits are found by considering $n$ fixed $(a \sim i \eta)$ and using the formulae [35]

$$
\begin{aligned}
& \lim _{a \rightarrow \infty} \Phi\left(a, b ;-\frac{x}{a}\right)=\Gamma(b) x^{(1-b) / 2} J_{b-1}(2 \sqrt{x}), \\
& \lim _{a \rightarrow \infty}\left[\Gamma(a+1-b) \Psi\left(a, b ; \frac{x}{a}\right)\right]=2 x^{(1-b) / 2} K_{b-1}(2 \sqrt{x})
\end{aligned}
$$

where $J_{\lambda}(\xi)$ and $K_{\lambda}(\xi)$ denote the Bessel and the modified Bessel function of the second kind [39], whose definitions in terms of confluent hypergeometric functions are [35]

$$
\begin{aligned}
& J_{\lambda}(t)=\frac{1}{\Gamma(\lambda+1)} e^{-i t}\left(\frac{t}{2}\right)^{\lambda} \Phi\left(\lambda+\frac{1}{2}, 2 \lambda+1 ; 2 i t\right), \\
& K_{\lambda}(t)=K_{-\lambda}(t)=\sqrt{\pi} e^{-t}(2 t)^{\lambda} \Psi\left(\lambda+\frac{1}{2}, 2 \lambda+1,2 t\right) .
\end{aligned}
$$

For using (76) when $i \eta \rightarrow \infty$ ( $n$ and $B_{2}$ fixed, $q=$ constant), first we make the approximations

$$
\begin{aligned}
& \Psi\left(n+i \eta+\frac{B_{2}}{2}, n+B_{2} ;-\frac{q z}{i \eta}\right) \approx \Psi\left(i \eta, n+B_{2} ;-\frac{q z}{i \eta}\right), \\
& \hat{\Phi}\left(n+i \eta+\frac{B_{2}}{2}, n+B_{2} ;-\frac{q z}{i \eta}\right) \approx \hat{\Phi}\left(i \eta, n+B_{2} ;-\frac{q z}{i \eta}\right) .
\end{aligned}
$$

In this manner we find the first set of solutions written in Eq. (178a). Notice that, although this is a formal derivation, the solutions can be tested by inserting them into Eq. (74), as shown in Appendix A.

First set. These solutions admit the Leaver limit. If $B_{2}+B_{1} / z_{0}=1$, the first and the fourth sets of solutions are equal to one another.

$$
\begin{aligned}
& U_{1}^{0}(z)=\sum_{n=0}^{\infty} b_{n}^{(1)}\left(z-z_{0}\right)^{n} \\
& U_{1}^{\infty}(z)=z^{\left(1-B_{2}\right) / 2} \sum_{n=0}^{\infty} c_{n}^{(1)}(i \sqrt{q z})^{-n} K_{n+B_{2}-1}(2 i \sqrt{q z}) \\
& U_{1}(z)=z^{\left(1-B_{2}\right) / 2} \sum_{n=0}^{\infty} c_{n}^{(1)}(\sqrt{q z})^{-n} J_{n+B_{2}-1}(2 \sqrt{q z})
\end{aligned}
$$

where the recurrence relations for $b_{n}^{(1)}$ and $c_{n}^{(1)}$ are $\left(b_{-1}^{(1)}=c_{-1}^{(1)}=0\right)$

$$
z_{0}\left(n+B_{2}+\frac{B_{1}}{z_{0}}\right)(n+1) b_{n+1}^{(1)}+\left[n\left(n+B_{2}-1\right)+B_{3}\right] b_{n}^{(1)}+q b_{n-1}^{(1)}=0,
$$

and

$$
(n+1) c_{n+1}^{(1)}+\left[n\left(n+B_{2}-1\right)+B_{3}\right] c_{n}^{(1)}+q z_{0}\left(n+B_{2}+\frac{B_{1}}{z_{0}}-1\right) c_{n-1}^{(1)}=0 .
$$

The coefficients $b_{n}^{(1)}$ and $c_{n}^{(1)}$ are again connected by Eq. (34). From this first set of solutions we obtain the others by applying the previous rules $T_{1}$ and $T_{2}$ according to relations (32). 
Second set. These solutions do not admit the Leaver limit. If $B_{2}+B_{1} / z_{0}=1$, the second and the third sets are equal to each other.

$$
\begin{aligned}
& U_{2}^{0}(z)=z^{1+\left(B_{1} / z_{0}\right)} \sum_{n=0}^{\infty} b_{n}^{(2)}\left(z-z_{0}\right)^{n}, \\
& U_{2}^{\infty}(z)=z^{\left(1-B_{2}\right) / 2} \sum_{n=0}^{\infty} c_{n}^{(2)}(i \sqrt{q z})^{-n} K_{n+1+B_{2}+\frac{2 B_{1}}{z_{0}}}(2 i \sqrt{q z}), \\
& U_{2}(z)=z^{\left(1-B_{2}\right) / 2} \sum_{n=0}^{\infty} c_{n}^{(2)}(\sqrt{q z})^{-n} J_{n+1+B_{2}+\frac{2 B_{1}}{z_{0}}}(2 \sqrt{q z}),
\end{aligned}
$$

where the recurrence relations are given by

$$
\begin{aligned}
& z_{0}\left(n+B_{2}+\frac{B_{1}}{z_{0}}\right)(n+1) b_{n+1}^{(2)}+ \\
& {\left[n\left(n+1+B_{2}+\frac{2 B_{1}}{z_{0}}\right)+\left(1+\frac{B_{1}}{z_{0}}\right)\left(B_{2}+\frac{B_{1}}{z_{0}}\right)+B_{3}\right] b_{n}^{(2)}+q b_{n-1}^{(2)}=0}
\end{aligned}
$$

and

$$
\begin{aligned}
& (n+1) c_{n+1}^{(2)}+\left[n\left(n+1+B_{2}+\frac{2 B_{1}}{z_{0}}\right)+\left(1+\frac{B_{1}}{z_{0}}\right)\left(B_{2}+\frac{B_{1}}{z_{0}}\right)+B_{3}\right] c_{n}^{(2)}+ \\
& q z_{0}\left(n+B_{2}+\frac{B_{1}}{z_{0}}-1\right) c_{n-1}^{(2)}=0 .
\end{aligned}
$$

The relation between $b_{n}^{(2)}$ and $c_{n}^{(2)}$ is given by Eq. (36).

Third set. These solutions admit Leaver limit.

$$
\begin{aligned}
& U_{3}^{0}(z)=z^{1+\frac{B_{1}}{z_{0}}}\left(z-z_{0}\right)^{1-B_{2}-\frac{B_{1}}{z_{0}}} \sum_{n=0}^{\infty} b_{n}^{(3)}\left(z-z_{0}\right)^{n}, \\
& U_{3}^{\infty}(z)=z^{\frac{B_{2}-1}{2}+\frac{B_{1}}{z_{0}}}\left(z-z_{0}\right)^{1-B_{2}-\frac{B_{1}}{z_{0}}} \sum_{n=0}^{\infty} c_{n}^{(3)}(i \sqrt{q z})^{-n} K_{n+3-B_{2}}(2 i \sqrt{q z}), \\
& U_{3}(z)=z^{\frac{B_{2}-1}{2}+\frac{B_{1}}{z_{0}}}\left(z-z_{0}\right)^{1-B_{2}-\frac{B_{1}}{z_{0}}} \sum_{n=0}^{\infty} c_{n}^{(3)}(\sqrt{q z})^{-n} J_{n+3-B_{2}}(2 \sqrt{q z}),
\end{aligned}
$$

with the recurrence relations

$$
z_{0}\left(n+2-B_{2}-\frac{B_{1}}{z_{0}}\right)(n+1) b_{n+1}^{(3)}+\left[n\left(n+3-B_{2}\right)+2-B_{2}+B_{3}\right] b_{n}^{(3)}+q b_{n-1}^{(3)}=0
$$

and

$$
(n+1) c_{n+1}^{(3)}+\left[n\left(n+3-B_{2}\right)+2-B_{2}+B_{3}\right] c_{n}^{(3)}+q z_{0}\left(n+1-B_{2}-\frac{B_{1}}{z_{0}}\right) c_{n-1}^{(3)}=0
$$

Eq. (38) affords the relations between $b_{n}^{(3)}$ and $c_{n}^{(3)}$. 
Fourth set. There is no limit for $z_{0} \rightarrow 0$.

$$
\begin{aligned}
& U_{4}^{0}(z)=\left(z-z_{0}\right)^{1-B_{2}-\frac{B_{1}}{z_{0}}} \sum_{n=0}^{\infty} b_{n}^{(4)}\left(z-z_{0}\right)^{n}, \\
& U_{4}^{\infty}(z)=z^{\frac{B_{1}}{z_{0}}+\frac{B_{2}}{2}-\frac{1}{2}}\left(z-z_{0}\right)^{1-B_{2}-\frac{B_{1}}{z_{0}}} \sum_{n=0}^{\infty} c_{n}^{(4)}(i \sqrt{q z})^{-n} K_{n+1-B_{2}-\frac{2 B_{1}}{z_{0}}}(2 i \sqrt{q z}), \\
& U_{4}(z)=z^{\frac{B_{1}}{z_{0}}+\frac{B_{2}}{2}-\frac{1}{2}}\left(z-z_{0}\right)^{1-B_{2}-\frac{B_{1}}{z_{0}}} \sum_{n=0}^{\infty} c_{n}^{(4)}(\sqrt{q z})^{-n} J_{n+1-B_{2}-\frac{2 B_{1}}{z_{0}}}(2 \sqrt{q z})
\end{aligned}
$$

with the recurrence relations

$$
\begin{aligned}
& z_{0}\left(n+2-B_{2}-\frac{B_{1}}{z_{0}}\right)(n+1) b_{n+1}^{(4)}+ \\
& {\left[n\left(n+1-B_{2}-\frac{2 B_{1}}{z_{0}}\right)+\frac{B_{1}}{z_{0}}\left(B_{2}+\frac{B_{1}}{z_{0}}-1\right)+B_{3}\right] b_{n}^{(4)}+q b_{n-1}^{(4)}=0}
\end{aligned}
$$

and

$$
\begin{aligned}
& (n+1) c_{n+1}^{(4)}+\left[n\left(n+1-B_{2}-\frac{2 B_{1}}{z_{0}}\right)+\frac{B_{1}}{z_{0}}\left(B_{2}+\frac{B_{1}}{z_{0}}-1\right)+B_{3}\right] c_{n}^{(4)}+ \\
& q z_{0}\left(n+1-B_{2}-\frac{B_{1}}{z_{0}}\right) c_{n-1}^{(4)}=0 .
\end{aligned}
$$

Now the coefficients $b_{n}^{(4)}$ and $c_{n}^{(4)}$ are connected by Eq. (40).

For the aforementioned wave equation which arises from the separation of variables of the Laplace-Beltrami operator for a scalar field on the Eguchi-Hanson space, Malmendier [19] has also found a solution in power series whose coefficients satisfy three-term recurrence relations. However, as a solution convergent in the neighborhood of $z=\infty$, he has proposed a subnormal Thomé solution with four-term recurrence relations, in contrast with the above solutions in series of Bessel functions.

\section{B. Mathieu equation and Lindemann-Stieltjes' solutions}

By inserting

$$
z_{0}=1, B_{1}=-1 / 2, B_{2}=1, z=\cos ^{2}(\sigma u), W(u)=U(z),
$$

into Eq. (74) we obtain the Mathieu equation

$$
\frac{d^{2} W}{d u^{2}}+\sigma^{2}\left[2 q-4 B_{3}-2 q \cos (2 \sigma u)\right] W=0 .
$$

Then, solutions for the Mathieu equation are obtained by making

$$
\left.\begin{array}{l}
W(u)=U(z), \quad z=\cos ^{2}(\sigma u),(\sigma=1, i), \\
z_{0}=1, B_{1}=-1 / 2, B_{2}=1, B_{3}=\frac{1}{4}[2 q-a], q=k^{2},
\end{array}\right\} \begin{aligned}
& \text { Mathieu Eq. as Whittaker- } \\
& \text { Ince limit of the GSWE }
\end{aligned}
$$

where $\sigma=1$ for the Mathieu equation, $\sigma=i$ for the modified Mathieu equation and $U(z)$ are the solutions for the Whittaker-Ince limit of the GSWE, Sec. IV A. Amongst the following solutions, 
included in the $W_{i}^{0}(u)$, are solutions found by Lindmann and Stieltjes, which are even or odd and have period $\pi$ or $2 \pi$ unlike the solutions of Sections II C and III C. As mentioned at the beginning of Sec II A, the following solutions may also be generated by letting $\xi$ and $p$ to tend respectively to zero and infinity so that $p \xi=2 k^{2}$ in the solutions of Sec. II B to WHE (11) $(\kappa=\sigma)$, that is, by following the original proposal of Whittaker and Ince [2, 3, 23].

First set. Even solutions. The solutions $W_{1}^{0}$ and $W_{1}$ have period $\pi$ if $\sigma=1$ since $J_{n}(-x)=$ $(-1)^{n} J_{n}(x)$.

$$
\begin{array}{ll}
W_{1}^{0}(u)=\sum_{n=0}^{\infty} \frac{(-1)^{n} c_{n}^{(1)}}{\Gamma[n+(1 / 2)]} \sin ^{2 n}(\sigma u), & |\cos (\sigma u)|<\infty, \\
W_{1}^{\infty}(u)=\sum_{n=0}^{\infty} c_{n}^{(1)}[i k \cos (\sigma u)]^{-n} K_{n}[2 i k \cos (\sigma u)], & |\cos (\sigma u)|>1, \\
W_{1}(u)=\sum_{n=0}^{\infty} c_{n}^{(1)}[k \cos (\sigma u)]^{-n} J_{n}[2 k \cos (\sigma u)], & \forall u .
\end{array}
$$

The recurrence relations are

$$
(n+1) c_{n+1}^{(1)}+\left(n^{2}+\frac{k^{2}}{2}-\frac{a}{4}\right) c_{n}^{(1)}+k^{2}\left(n-\frac{1}{2}\right) c_{n-1}^{(1)}=0 .
$$

Second set. Even solutions. If $\sigma=1 W_{2}^{0}$ and $W_{2}$ have period $2 \pi$.

$$
\begin{array}{ll}
W_{2}^{0}(u)=\cos (\sigma u) \sum_{n=0}^{\infty} \frac{(-1)^{n} b_{n}^{(1)}}{\Gamma[n+(1 / 2)]} \sin ^{2 n}(\sigma u), & |\cos (\sigma u)|<\infty, \\
W_{2}^{\infty}(u)=\sum_{n=0}^{\infty} c_{n}^{(1)}[i k \cos (\sigma u)]^{-n} K_{n+1}[2 i k \cos (\sigma u)], & |\cos (\sigma u)|>1, \\
W_{2}(u)=\sum_{n=0}^{\infty} c_{n}^{(1)}[k \cos (\sigma u)]^{-n} J_{n+1}[2 k \cos (\sigma u)], & \forall u,
\end{array}
$$

with the recurrence relations

$$
(n+1) c_{n+1}^{(2)}+\left[n(n+1)+\frac{1}{4}+\frac{k^{2}}{2}-\frac{a}{4}\right] c_{n}^{(2)}+k^{2}\left(n-\frac{1}{2}\right) c_{n-1}^{(2)}=0
$$

Third set. Odd solutions. If $\sigma=1, W_{3}^{0}$ and $W_{3}$ have period $\pi$.

$$
\begin{array}{ll}
W_{3}^{0}(u)=\cos (\sigma u) \sum_{n=0}^{\infty} \frac{(-1)^{n} c_{n}^{(3)}}{\Gamma[n+(1 / 2)]} \sin ^{2 n+1}(\sigma u), & |\cos (\sigma u)|<\infty, \\
W_{3}^{\infty}(u)=\tan (\sigma u) \sum_{n=0}^{\infty} c_{n}^{(3)}[i k \cos (\sigma u)]^{-n} K_{n+2}[2 i k \cos (\sigma u)], & |\cos (\sigma u)|>1, \\
W_{3}(u)=\tan (\sigma u) \sum_{n=0}^{\infty} c_{n}^{(3)}[k \cos (\sigma u)]^{-n} J_{n+2}[2 k \cos (\sigma u)], & \mid \forall u .
\end{array}
$$


The recurrence relations are

$$
(n+1) c_{n+1}^{(3)}+\left[n(n+2)+1+\frac{k^{2}}{2}-\frac{a}{4}\right] c_{n}^{(3)}+k^{2}\left(n+\frac{1}{2}\right) c_{n-1}^{(3)}=0 .
$$

Fourth set. Odd solutions. For $\sigma=1, W_{4}^{0}(z)$ and $W_{4}(z)$ have period $2 \pi$.

$$
\begin{array}{ll}
W_{4}^{0}(u)=\sum_{n=0}^{\infty} \frac{(-1)^{n} b_{n}^{(4)}}{\Gamma[n+(3 / 2)]} \sin ^{2 n+1}(\sigma u), & |\cos (\sigma u)|<\infty, \\
W_{4}^{\infty}(u)=\tan (\sigma u) \sum_{n=0}^{\infty} c_{n}^{(4)}[i k \cos (\sigma u)]^{-n} K_{n+1}[2 i k \cos (\sigma u)], & |\cos (\sigma u)|>1, \\
W_{4}(u)=\tan (\sigma u) \sum_{n=0}^{\infty} c_{n}^{(4)}[k \cos (\sigma u)]^{-n} J_{n+1}[2 k \cos (\sigma u)], & \forall u .
\end{array}
$$

The recurrence relations are

$$
(n+1) c_{n+1}^{(4)}+\left[n(n+1)+\frac{1}{4}+\frac{k^{2}}{2}-\frac{a}{4}\right] c_{n}^{(4)}+k^{2}\left(n+\frac{1}{2}\right) c_{n-1}^{(4)}=0 .
$$

From the preceding solutions other expansions may be obtained by the substitutions

$$
u \rightarrow u+\frac{\pi}{2 \sigma} \text { and } k \rightarrow i k,
$$

which plays the role of the transformation $T_{4}$ given in Eq. (75). The solutions $W_{1}^{0}, W_{2}^{0}$ and those resulting from these by means of (87), if expressed in terms of $z\left[z=\cos ^{2}(\sigma u)\right]$, are the ones proposed by Lindemann and Stieltjes [29, 30] but here to each of these now correspond two solutions in series of Bessel functions.

\section{Whittaker-Ince's limits for the DCHE}

The solutions for the Whittaker-Ince limit (9) of the DCHE, that is, for

$$
z^{2} \frac{d^{2} U}{d z^{2}}+\left(B_{1}+B_{2} z\right) \frac{d U}{d z}+\left(B_{3}+q z\right) U=0,\left(q \neq 0, B_{1} \neq 0\right)
$$

may be obtained from the solutions for the DCHE, given Sec. III A, by letting $\omega \rightarrow 0$ and $\eta \rightarrow \infty$ such that $2 \eta \omega=-q$. However, at this stage, it is simpler to take the Leaver limit $z_{0} \rightarrow 0$ of the first and third sets of solutions given in Sec. IV A. As in the cases of the Mathieu equation there is no finite-series solution.

First set. If we put $b_{n}^{(1)}=c_{n}^{(1)} /\left(B_{1}\right)^{n}$ in the limits of solutions (78a), we find

$$
\begin{aligned}
& U_{1}^{0}(z)=\sum_{n=0}^{\infty} c_{n}^{(1)}\left(\frac{z}{B_{1}}\right)^{n}, \\
& U_{1}^{\infty}(z)=z^{\left(1-B_{2}\right) / 2} \sum_{n=0}^{\infty} c_{n}^{(1)}(i \sqrt{q z})^{-n} K_{n+B_{2}-1}(2 i \sqrt{q z}), \\
& U_{1}(z)=z^{\left(1-B_{2}\right) / 2} \sum_{n=0}^{\infty} c_{n}^{(1)}(\sqrt{q z})^{-n} J_{n+B_{2}-1}(2 \sqrt{q z}),
\end{aligned}
$$


where the recurrence relations for $c_{n}^{(1)}$ are $\left(c_{-1}^{(1)}=0\right)$

$$
(n+1) c_{n+1}^{(1)}+\left[n\left(n+B_{2}-1\right)+B_{3}\right] c_{n}^{(1)}+q B_{1} c_{n-1}^{(1)}=0 .
$$

Second set. In the limit of third set of solutions given in Sec. IV A we take

$$
b_{n}^{(3)}=c_{n}^{(2)} /\left(-B_{1}\right)^{n}, \quad \lim _{z_{0} \rightarrow 0}\left(1-\frac{z_{0}}{z}\right)^{-B_{1} / z_{0}}=e^{B_{1} / z} .
$$

Then, we obtain

$$
\begin{aligned}
& U_{2}^{0}(z)=z^{2-B_{2}} e^{B_{1} / z} \sum_{n=0}^{\infty} c_{n}^{(2)}\left(-\frac{z}{B_{1}}\right)^{n}, \\
& U_{2}^{\infty}(z)=z^{\left(1-B_{2}\right) / 2} e^{B_{1} / z} \sum_{n=0}^{\infty} c_{n}^{(2)}(i \sqrt{q z})^{-n} K_{n+3-B_{2}}(2 i \sqrt{q z}), \\
& U_{2}(z)=z^{\left(1-B_{2}\right) / 2} e^{B_{1} / z} \sum_{n=0}^{\infty} c_{n}^{(2)}(\sqrt{q z})^{-n} J_{n+3-B_{2}}(2 \sqrt{q z}),
\end{aligned}
$$

with the recurrence relations

$$
(n+1) c_{n+1}^{(2)}+\left[n\left(n+3-B_{2}\right)+2-B_{2}+B_{3}\right] c_{n}^{(2)}-q B_{1} c_{n-1}^{(2)}=0 .
$$

As we see, in the present context, it is almost trivial to obtain solutions for Eq. (88).

\section{Solutions with a phase parameter}

In this section we make some comments on solutions in which a phase parameter $\nu$ was introduced to assure the series convergence if there is no free constant in the GSWE. Such solutions were partly studied elsewhere [1, 10, 25, 26] but here we provide some more informations useful in applications. These solutions, which have been used in the study of the Teukolsky equations in Kerr spacetimes [25, 26, 27, 28, will as well be taken as a possible starting-point to find new solutions to the general Heun equation (Appendix B).

Instead of three solutions as in Ref. 10, we consider a basic set containing four solutions, two given by expansions in series of hypergeometric functions $F(a, b ; c ; y)$ and two by expansions in series of confluent hypergeometric functions. These solutions, like the ones of Sec. II, admit both the Leaver and the Whittaker-Ince limits, but the last limit is not regarded here because it is the same as in Ref. 44, apart from the fact that now we have four solutions instead of two.

In the first place we remark that there are several physical problems ruled by differential equations without any free constant, that is, requiring a phase parameter. For example, all the constants appearing in the GSWEs responsible by the time-dependence of Klein-Gordon and Dirac test-fields in some curved spacetimes are determined from the spatial part of the wavefunction 10, 40, 41, 42. Similarly, in the radial Schrödinger equation for the scattering of electrons by a finite dipole [1] or by polarizable targets [4, 43, 44, 45] there is no disposable constant, since all the parameters which characterize the target and the incident particles are known.

In the second place, solutions with a phase parameter $\nu$ are given by doubly infinite series $(-\infty<n<\infty)$ and, if $b_{n}$ denotes the series coefficients, then the recurrence relations have the general form

$$
\alpha_{n} b_{n+1}+\beta_{n} b_{n}+\gamma_{n} b_{n-1}=0,(-\infty<n<\infty)
$$


where $\alpha_{n}, \beta_{n}, \gamma_{n}$ and $b_{n}$ depend on $\nu$ and on the parameters of the GSWE. These recurrence relations give the characteristic equation [1]

$$
\beta_{0}=\frac{\alpha_{-1} \gamma_{0}}{\beta_{-1}-} \frac{\alpha_{-2} \gamma_{-1}}{\beta_{-2}-} \frac{\alpha_{-3} \gamma_{-2}}{\beta_{-3}-} \cdots+\frac{\alpha_{0} \gamma_{1}}{\beta_{1}-} \frac{\alpha_{1} \gamma_{2}}{\beta_{2}-} \frac{\alpha_{2} \gamma_{3}}{\beta_{3}-} \cdots,
$$

wherefrom $\nu$ must be determined. For a specific set of solutions we add a superscript in each of these quantities.

Moreover, as we are going to explain, solutions with a phase parameter are also important because they can be used in two different manners to obtain solutions for equations having a free constant.

\section{A. Spheroidal equation}

The sets of solutions are denoted by by $\left(U_{i \nu}^{0}, \hat{U}_{i \nu}^{\infty}, U_{i \nu}^{\infty}, \widetilde{U}_{i \nu}^{\infty}\right)(i=1,2)$ : the solutions $U_{i \nu}^{0}$ converge for any finite $z$ and the others converge for $|z|>\left|z_{0}\right|$. These solutions were obtained by integrating the the GSWE [1, 10, 25, 26], except $\hat{U}_{i \nu}^{\infty}$ which is derived from solutions of the general Heun equation in Appendix B. We write only the first set of solutions (the second set is obtained from the first by using the transformation rule $T_{2}$ ), namely,

$$
\begin{aligned}
U_{1 \nu}^{0}(z)= & e^{i \omega z} \sum_{n=-\infty}^{\infty} b_{n}^{(1)} F\left(\frac{B_{2}}{2}-n-\nu-1, n+\nu+\frac{B_{2}}{2} ; B_{2}+\frac{B_{1}}{z_{0}} ; \frac{z_{0}-z}{z_{0}}\right), \\
\hat{U}_{1 \nu}^{\infty}(z)= & e^{i \omega z} \sum_{n=-\infty}^{\infty} \hat{b}_{n}^{(1)}\left(\frac{z_{0}-z}{z_{0}}\right)^{-n-\nu-\frac{B_{2}}{2}} \times \\
& \widetilde{F}\left(n+\nu+\frac{B_{2}}{2}, n+\nu+1-\frac{B_{2}}{2}-\frac{B_{1}}{z_{0}} ; 2 n+2 \nu+2 ; \frac{z_{0}}{z_{0}-z}\right), \\
U_{1 \nu}^{\infty}(z)= & e^{i \omega z} z^{1-\left(B_{2} / 2\right)} \sum_{n=-\infty}^{\infty} b_{n}^{(1)}(-2 i \omega z)^{n+\nu} \Psi(n+\nu+1+i \eta, 2 n+2 \nu+2 ;-2 i \omega z), \\
\widetilde{U}_{1 \nu}^{\infty}(z)= & e^{i \omega z} z^{1-\left(B_{2} / 2\right)} \sum_{n=-\infty}^{\infty} b_{n}^{(1)}(2 i \omega z)^{n+\nu} \widetilde{\Phi}(n+\nu+1+i \eta, 2 n+2 \nu+2 ;-2 i \omega z),
\end{aligned}
$$

where the functions $\widetilde{F}(a, b ; c ; y)$ and $\widetilde{\Phi}(a, b ; y)$ are defined by means of

$$
\widetilde{F}(a, b ; c ; y)=\frac{F(a, b ; c ; y)}{\Gamma(c)}, \quad \widetilde{\Phi}(a, b ; z)=\frac{\Gamma(b-a)}{\Gamma(b)} \Phi(a, b ; z)
$$

and the coefficients $\hat{b}_{n}^{(1)}$ are connected with $b_{n}^{(1)}$ through

$$
\hat{b}_{n}^{(1)}:=(-1)^{n} \Gamma\left(n+\nu+2-\frac{B_{2}}{2}\right) \Gamma\left(n+\nu+1-\frac{B_{1}}{z_{0}}-\frac{B_{2}}{2}\right) b_{n}^{(1)}, \quad \text { if } z_{0} \neq 0 .
$$

The coefficients of the recurrence relations (91a) for $b_{n}^{(1)}$ are

$$
\begin{aligned}
& \alpha_{n}^{(1)}=i \omega z_{0} \frac{\left(n+\nu+2-\frac{B_{2}}{2}\right)\left(n+\nu+1-\frac{B_{2}}{2}-\frac{B_{1}}{z_{0}}\right)(n+\nu+1-i \eta)}{2(n+\nu+1)\left(n+\nu+\frac{3}{2}\right)} \\
& \beta_{n}^{(1)}=-B_{3}-\eta \omega z_{0}-\left(n+\nu+1-\frac{B_{2}}{2}\right)\left(n+\nu+\frac{B_{2}}{2}\right)-\frac{\eta \omega z_{0}\left(\frac{B_{2}}{2}-1\right)\left(\frac{B_{2}}{2}+\frac{B_{1}}{z_{0}}\right)}{(n+\nu)(n+\nu+1)}, \\
& \gamma_{n}^{(1)}=-i \omega z_{0} \frac{\left(n+\nu+\frac{B_{2}}{2}-1\right)\left(n+\nu+\frac{B_{2}}{2}+\frac{B_{1}}{z_{0}}\right)(n+\nu+i \eta)}{2\left(n+\nu-\frac{1}{2}\right)(n+\nu)} .
\end{aligned}
$$


Note that the hypergeometric functions in $U_{1 \nu}^{0}$ are well defined only if $B_{2}+\left(B_{1} / z_{0}\right) \neq 0,-1,-2, \cdots$, while in $U_{2 \nu}^{0}$ we would have the restriction $B_{2}+\left(B_{1} / z_{0}\right) \neq 2,3,4, \cdots$.

In these solutions, $\nu$ cannot be integer or half-integer in order to prevent two dependent terms (for different values of $n$ ) inside the summations. Suppose, on the contrary, that $2 \nu+1=N$ in $U_{1 \nu}^{0}$, for some integer $N$. Then, for $n=n_{1}\left(\forall n_{1}\right)$ and $n=-N-n_{1}$, the hypergeometric functions in $U_{1 \nu}^{0}$ would be equal on account of their symmetry with respect to the two first parameters. One may show that this is the same for the other solutions. In fact, integer or half-integer values for $\nu$ would lead to infinity coefficients in the recurrence relations for $b_{n}^{(1)}$.

Now we consider the two possibilities for getting solutions without phase parameter from the ones with a phase parameter. The first consists in breaking off the series by taking $n \geq 0$ in which case the parameter $\nu$ is given in terms of the parameters of the differential equation [4, 10]. In particular, the truncation of $U_{1}^{0}$ gives an expansion in series of Jacobi polynomials which includes the Baber-Hassé solutions (associated Legendre polynomials) for the angular equation of the twocenter problem of quantum mechanics [24] as well as the Fackerell-Crossman solutions for angular Teukolsky equations [46]. In this regard, we must do the following correction in Ref. [10]: the truncated expansions in series of regular confluent hypergeometric functions converge for $|z|>\left|z_{0}\right|$, rather than for any $z$ as stated there. Therefore, such solutions are not suitable to solve an angular equation because in this case $|z| \leq\left|z_{0}\right|$.

The other possibility gives solutions in terms of doubly infinite series $(-\infty<n<\infty)$. These are obtained by ascribing any convenient value to $\nu$, excepting integer or half-integer values. Such solutions exhibit properties that may be quite different from the properties of the one-sided series $(n \geq 0)$, as illustrated by the following solution for WHE which we derive from a solution for the GSWE. Proceeding as in Sec. II B and using the relation [36]

$$
F\left(-a, a ; 1 / 2 ; \sin ^{2} u\right)=\cos (2 a u),
$$

we find that the solution $U_{1 \nu}^{0}(z)$ affords an even solution for the WHE, namely,

$$
W_{1 \nu}^{0}(u)=e^{(\xi / 2) \cos ^{2}(\kappa u)} \sum_{n=-\infty}^{\infty} b_{n} \cos [(2 n+2 \nu+1) \kappa u]
$$

where the recurrence relations for $b_{n}$ are $(-\infty<n<\infty)$

$$
\xi\left(n+\nu+\frac{1}{2}-\frac{p}{2}\right) b_{n+1}+\left[\vartheta-(2 n+2 \nu+1)^{2}\right] b_{n}-\xi\left(n+\nu+\frac{1}{2}+\frac{p}{2}\right) b_{n-1}=0 .
$$

Now, if there is a free parameter in the differential equation, we can choose any admissible value for $\nu$ and, in particular,

$$
2 \nu+1=l / m, \quad \kappa=1,
$$

where $l$ and $m$ are integers prime to one another, so that $1<l<m$. Then, we find the following two-sided Ince solution, $W_{1}^{I}(u)$, for the WHE [3]

$$
W_{1}^{I}(u)=e^{\frac{\xi}{2} \cos ^{2}(u)} \sum_{n=-\infty}^{\infty} b_{n}^{(1)} \cos \left[\left(2 n+\frac{l}{m}\right) u\right]
$$

having period $2 \pi m, m>1$, in opposition to the solutions obtained from the truncated solutions [10] (period $\pi$ or $2 \pi)$. An odd solution may also be found from the $U_{2 \nu}^{0}(z)$ of the (omitted) second set of solutions. 
Although this example is concerned only with the periodicity and parity of solutions for the Whittaker-Hill equation, the analysis can be extended to other aspects of the GSWE and their limiting cases. Next we use two-sided solutions similar to the above one to complete the solutions of the Schrödinger equation considered in section III.

\section{B. Double-confluent Heun and Schrödinger equations}

From the previous solutions there is no difficulty in finding solutions for the DCHE by means of the Leaver limit, provided that we use the formulas [35]

$$
\begin{aligned}
& \lim _{c \rightarrow \infty} F\left(a, b ; c ; 1-\frac{c}{y}\right)=y^{a} \Psi(a, a+1-b ; y), \\
& \lim _{b \rightarrow \infty} F\left(a, b ; c ; \frac{y}{b}\right)=\Phi(a, c ; y) .
\end{aligned}
$$

We find the Leaver solutions [1, 10]

$$
\begin{aligned}
& U_{1 \nu}^{0}(z)=e^{i \omega z} z^{-\frac{B_{2}}{2}} \sum_{n=-\infty}^{\infty} b_{n}^{(1)}\left(\frac{B_{1}}{z}\right)^{n+\nu} \Psi\left(n+\nu+\frac{B_{2}}{2}, 2 n+2 \nu+2 ; \frac{B_{1}}{z}\right), \\
& \hat{U}_{1 \nu}^{\infty}(z)=e^{i \omega z} z^{-\frac{B_{2}}{2}} \sum_{n=-\infty}^{\infty} b_{n}^{(1)}\left(-\frac{B_{1}}{z}\right)^{n+\nu} \tilde{\Phi}\left(n+\nu+\frac{B_{2}}{2}, 2 n+2 \nu+2 ; \frac{B_{1}}{z}\right), \\
& U_{1 \nu}^{\infty}(z)=e^{i \omega z} z^{1-\frac{B_{2}}{2}} \sum_{n=-\infty}^{\infty} b_{n}^{(1)}(-2 i \omega z)^{n+\nu} \Psi(n+\nu+1+i \eta, 2 n+2 \nu+2 ;-2 i \omega z), \\
& \widetilde{U}_{1 \nu}^{\infty}(z)=e^{i \omega z} z^{1-\frac{B_{2}}{2}} \sum_{n=-\infty}^{\infty} b_{n}^{(1)}(2 i \omega z)^{n+\nu} \tilde{\Phi}(n+\nu+1+i \eta, 2 n+2 \nu+2 ;-2 i \omega z),
\end{aligned}
$$

with the following coefficients in the recurrence relations (91a) for $b_{n}^{(1)}$ :

$$
\begin{aligned}
& \alpha_{n}^{(1)}=i \omega B_{1} \frac{\left(n+\nu+2-\frac{B_{2}}{2}\right)(n+\nu+1-i \eta)}{2(n+\nu+1)\left(n+\nu+\frac{3}{2}\right)} \\
& \beta_{n}^{(1)}=B_{3}-\frac{1}{4}\left(B_{2}-1\right)^{2}+\left(n+\nu+\frac{1}{2}\right)^{2}+\frac{\eta \omega B_{1}\left(\frac{B_{2}}{2}-1\right)}{(n+\nu)(n+\nu+1)} \\
& \gamma_{n}^{(1)}=i \omega B_{1} \frac{\left(n+\nu+\frac{B_{2}}{2}-1\right)(n+\nu+i \eta)}{2(n+\nu)\left(n+\nu-\frac{1}{2}\right)} .
\end{aligned}
$$

These four solutions result as the Leaver limits of solutions for the GSWE because in Sec. VI.A we have chosen a set formed by four solutions. They can also be obtained by starting with the two expansions in series of confluent hypergeometric functions for the GSWE and, then, using a symmetry of the DCHE [1, 10].

Now we use these solutions of the DCHE to find infinite-series solutions for the quasi-exactly solvable double-Morse potential discussed in section III. For this is necessary to match two solutions having different regions of convergence, a procedure which has been employed to find solutions for the radial Teukolsky equations in Kerr backgrounds [25, 26, 27, 28]. In this manner, the missing portion of the energy spectrum may be obtained. 
By inserting the previous $U_{1 \nu}^{0}$ and $U_{1 \nu}^{\infty}$ into Eq. (61) and using the parameters given in Eq. (62), we get, respectively,

$$
\begin{aligned}
& \phi_{1 \nu}^{0}(z)=\exp \left[-\frac{B}{4}\left(z+\frac{1}{z}\right)\right] \sum_{n=-\infty}^{\infty} b_{n}^{(1)}\left(\frac{B}{2 z}\right)^{n+\nu+\frac{1}{2}} \Psi\left(n+\nu+\frac{1}{2}+\frac{C}{2}-s, 2 n+2 \nu+2 ; \frac{B}{2 z}\right), \\
& \phi_{1 \nu}^{\infty}(z)=\exp \left[-\frac{B}{4}\left(z+\frac{1}{z}\right)\right] \sum_{n=-\infty}^{\infty} b_{n}^{(1)}\left(\frac{B z}{2}\right)^{n+\nu+\frac{1}{2}} \Psi\left(n+\nu+\frac{1}{2}-\frac{C}{2}-s, 2 n+2 \nu+2 ; \frac{B z}{2}\right),
\end{aligned}
$$

where in the recurrence relations (91a) for $b_{n}^{(1)}$ now we have $(B>0, C>0)$

$$
\begin{aligned}
& \alpha_{n}^{(1)}=-\frac{B^{2}}{16} \frac{\left(n+\nu+\frac{3}{2}-\frac{C}{2}+s\right)\left(n+\nu+\frac{3}{2}+\frac{C}{2}+s\right)}{(n+\nu+1)\left(n+\nu+\frac{3}{2}\right)} \\
& \beta_{n}^{(1)}=\mathcal{E}+\frac{B^{2}}{8}-\frac{C^{2}}{4}+\left(n+\nu+\frac{1}{2}\right)^{2}-\frac{B^{2}}{32} \frac{\left[C^{2}-(1+2 s)^{2}\right]}{(n+\nu)(n+\nu+1)}, \\
& \gamma_{n}^{(1)}=-\frac{B^{2}}{16} \frac{\left(n+\nu+\frac{C}{2}-\frac{1}{2}-s\right)\left(n+\nu-\frac{C}{2}-\frac{1}{2}-s\right)}{(n+\nu)\left(n+\nu-\frac{1}{2}\right)} .
\end{aligned}
$$

For infinite series, these solutions satisfy the regularity conditions (63) since we have $(B>0)$

$$
\begin{aligned}
& \lim _{z \rightarrow 0} \phi_{1 \nu}^{0}(z) \sim z^{\frac{C}{2}-s} \exp \left[-\frac{B}{4}\left(z+\frac{1}{z}\right)\right] \rightarrow 0, \\
& \lim _{z \rightarrow \infty} \phi_{1 \nu}^{\infty}(z) \sim z^{\frac{C}{2}+s} \exp \left[-\frac{B}{4}\left(z+\frac{1}{z}\right)\right] \rightarrow 0,
\end{aligned}
$$

where we have used the relation (27). However, in order to guarantee that in fact these are solutions given by infinite series, we must choose suitable values for the parameter $\nu$. Recalling that $\nu$ cannot be integer or half-integer and that $s$ is a non-negative integer or half-integer, we find that there are three different cases to be considered.

First case: $C$ is integer or half-integer. In this case we can take $\nu=1 / 3$ to find

$$
\begin{aligned}
& \alpha_{n}^{(1)}=-\frac{B^{2}}{16} \frac{\left(n+s+\frac{3}{2}+\frac{1}{3}-\frac{C}{2}\right)\left(n+s+\frac{3}{2}+\frac{1}{3}+\frac{C}{2}\right)}{\left(n+1+\frac{1}{3}\right)\left(n+\frac{1}{3}+\frac{3}{2}\right)}, \\
& \beta_{n}^{(1)}=\mathcal{E}+\frac{B^{2}}{8}-\frac{C^{2}}{4}+\left(n+\frac{1}{3}+\frac{1}{2}\right)^{2}-\frac{B^{2}}{32} \frac{\left[C^{2}-(1+2 s)^{2}\right]}{\left(n+\frac{1}{3}\right)\left(n+1+\frac{1}{3}\right)}, \\
& \gamma_{n}^{(1)}=-\frac{B^{2}}{16} \frac{\left(n-s-\frac{1}{2}+\frac{1}{3}+\frac{C}{2}\right)\left(n-s-\frac{1}{2}+\frac{1}{3}-\frac{C}{2}\right)}{\left(n+\frac{1}{3}\right)\left(n+\frac{1}{3}-\frac{1}{2}\right)} .
\end{aligned}
$$

Thence, the numerators of $\alpha_{n}^{(1)}$ and $\gamma_{n}^{(1)}$ do not vanish since $(1 / 3)+(C / 2)$ and $(1 / 3)-(C / 2)$ are not integer or half-integer. Therefore, we have a pair of solutions given by two-sided infinite series.

Second case: $C$ is not integer or half-integer, but $s$ is integer. In this case we can take

$$
\nu=\frac{C}{2}+1
$$

which is different of integer or half-integer. Then,

$$
\begin{aligned}
& \alpha_{n}^{(1)}=-\frac{B^{2}}{16} \frac{\left(n+s+\frac{5}{2}\right)\left(n+s+\frac{5}{2}+C\right)}{\left(n+2+\frac{C}{2}\right)\left(n+\frac{5}{2}+\frac{C}{2}\right)}, \\
& \beta_{n}^{(1)}=\mathcal{E}+\frac{B^{2}}{8}-\frac{C^{2}}{4}+\left(n+\frac{3}{2}+\frac{C}{2}\right)^{2}-\frac{B^{2}}{32} \frac{\left[C^{2}-(1+2 s)^{2}\right]}{\left(n+1+\frac{C}{2}\right)\left(n+2+\frac{C}{2}\right)}, \\
& \gamma_{n}^{(1)}=-\frac{B^{2}}{16} \frac{\left(n-s+\frac{1}{2}+C\right)\left(n-s+\frac{1}{2}\right)}{\left(n+1+\frac{C}{2}\right)\left(n+\frac{1}{2}+\frac{C}{2}\right)} .
\end{aligned}
$$


Thus the numerators of $\alpha_{n}^{(1)}$ and $\gamma_{n}^{(1)}$ cannot vanish and, once more, we have two-sided infinite series.

Third case: $C$ is not integer or half-integer, but $s$ is half-integer. In this case we can take

$$
\nu=\frac{C}{2}+\frac{1}{2}
$$

which again imply that the numerators of $\alpha_{n}^{(1)}$ and $\gamma_{n}^{(1)}$ cannot vanish since

$$
\begin{aligned}
& \alpha_{n}^{(1)}=-\frac{B^{2}}{16} \frac{(n+s+2)(n+s+2+C)}{\left(n+\frac{3}{2}+\frac{C}{2}\right)\left(n+2+\frac{C}{2}\right)}, \\
& \beta_{n}^{(1)}=\mathcal{E}+\frac{B^{2}}{8}-\frac{C^{2}}{4}+\left(n+1+\frac{C}{2}\right)^{2}-\frac{B^{2}}{32} \frac{\left[C^{2}-(1+2 s)^{2}\right]}{\left(n+\frac{1}{2}+\frac{C}{2}\right)\left(n+\frac{3}{2}+\frac{C}{2}\right)}, \\
& \gamma_{n}^{(1)}=-\frac{B^{2}}{16} \frac{(n-s+C)(n-s)}{\left(n+\frac{1}{2}+\frac{C}{2}\right)\left(n+\frac{C}{2}\right)} .
\end{aligned}
$$

Therefore, in principle this problem can be solved by matching the two double-sided solutions which converge over different ranges of the independent variable $z$. The energy spectrum can be obtained by solving the characteristic equation (91b). Then, for a given value of $s$, besides the energy levels provided by the quasi-polynomials solutions of Sec. III.A, there is the spectrum resulting from the present infinite-series solutions. In addition, for $C=0$, the first case affords solutions in terms of doubly infinite series for the symmetric double-Morse potential (WHE).

\section{Conclusions}

We have seen that the Leaver and Whittaker-Ince limits of the generalized spheroidal wave equation (GSWE) allow us to generate solutions for three other equations with different types of singular points. We have also obtained new solutions for the Mathieu and Whittaker-Hill equations by regarding these as particular cases of the GSWE and its limiting cases. As an application we have considered the Schrödinger equation with an asymmetric double-Morse potential.

First we have constructed a set of solutions to the GSWE in such a manner that the three solutions of each set converge over different regions but have series coefficients proportional to one another. Actually, this is an extention of an approach already used in a previous work [10] (revisited in Sec. VI), the difference being that in Sec. II the initial set of solutions is given by a series of ascending powers of $z-z_{0}$, due to Barber and Hassé, and two expansions in series of confluent hypergeometric functions. From the initial set other ones have been generated by means of transformation rules which reflect the symmetries of the GSWE. The importance of these rules can be appreciated by considering the properties of each set of solutions that we have found for the Mathieu and Whittaker-Hill equations.

From solutions of the GSWE, sets of solutions for the three limiting cases have been found following the diagram (10). Thus, the solutions for the double-confluent Heun equation (DCHE) have resulted by means of the Leaver limit. On the other hand, in the Whittaker-Ince limit for the GSWE and DCHE, the expansions in series of confluent hypergeometric functions have afforded expansions in series of Bessel functions as solutions for Eqs. (7) and (9).

In this process for generating solutions, the Barber-Hassé expansions have led to some known solutions for the DCHE, Mathieu and Whittaker-Hill equations, but these solutions are accompanied by two new solutions belonging to each set. Besides this, the Whittaker-Ince limit of the Barber-Hassé expansions has also given solutions which include as particular case the solution already proposed by Malmendier [19] to solve a wave equation which arises from separation of 
variables of the Laplace-Beltrami operator for a scalar field on the Eguchi-Hanson space. However, now we have found two expansions in series of Bessel functions, both of them convergent in the vicinity of $z=\infty$ and satisfying three-term recurrence relations for the series coefficients, in contrast with the solution proposed by Malmendier (four-term recurrence relations).

The properties of the solutions for the Mathieu and Whittaker-Hill equations depend on whether these solutions come from the GSWE or DCHE. We have seen that the unique solutions for the WHE which are even or odd and have period $\pi$ or $2 \pi$ result from the solutions for the GSWE (Sec. II B). The Whittaker-Ince limit transfer these properties to the solutions of the Mathieu equation given in Sec. IV B, each set including one of the Lindemann-Stieltjes solutions and two new solutions in series of Bessel functions. The latter solutions demand further investigation since they are different from the usual expansions in series of Bessel functions.

On the other hand, the solutions for WHE considered as a DCHE (Sec. III B) do not possess such properties of parity and periodicity (even or odd, period $\pi$ or $2 \pi$ ). The same is true for the solutions of the Mathieu equation when this last is treated as a particular case (not as WhittakerInce limit) of the WHE (Sec. II C) or as a particular case of the DCHE (Sec. III C). However, solutions for the Mathieu and Whittaker-Hill equations which do not present the usual properties of parity and periodicity may be important to solve initial value problems instead of boundary value problems [22].

Up to section $\mathrm{V}$ we have assumed that there is a free constant in the differential equation, and all the solutions are one-sided in the sense that the summation index $n$ runs from 0 to $\infty$. In this case, the free constant is determined from a characteristic equation which must be fulfilled in order to assure the series convergence. However, in section VI we have reconsidered a set of solutions in series of hypergeometric and confluent hypergeometric functions which converge when there is no free constant in the differential equation. In such solutions there is a parameter $\nu$, called phase or characteristic parameter [1, 21], which must be adjusted so that a characteristic equation is satisfied in order to guarantee the series convergence.

The solutions with the phase parameter $\nu$ are two-sided $(-\infty<n<\infty)$ and also admit both the Leaver and Whittaker-Ince limits. In addition, they allow us to find one-sided and two-sided solutions for an equation having a free parameter. The one-sided solutions are obtained by the restriction $n \geq 0$ in which case $\nu$ becomes determined in terms of other parameters of the equation [10]. The two-sided solutions are generated by choosing some of the admissible values for $\nu$, that is, some $\nu$ different from integer or half-integer. These two-sided solutions have been used in section VI B to find infinite-series solutions for the Schrödinger equation with an asymmetric double-Morse potential, by reducing that equation to a DCHE.

The asymmetric double-Morse potential considered here is a quasi-exactly solvable (QES) potential for which a part of the energy spectrum can be determined from the recurrence relations for the coefficients of finite-series eigensolutions, as the ones found in Sec II A. On the other hand, in Sec. VI B we have shown that, for a given value of the the parameter $s$, the other portion of the spectrum can be derived as solutions for a characteristic equation associated with eigenfunctions given by infinite two-sided series, provided that we accept to match two solutions which converge over different ranges of the independent variable. When the parameter $C$ vanishes, the asymmetric potential degenerate to a symmetric one, whereas the Schrödinger equation degenerate to a WHE which has already been solved as a GSWE [10]. Therefore, this symmetric case is an instance of a WHE which can be regarded both as a DCHE and a GSWE.

On the other hand, for the trigonometric and hyperbolic QES potentials given by Usheveridze [8], the Schrödinger equation can be transformed into GSWEs [38]. Thus, the solutions for the GSWE given in this paper may be used to verify if it is possible to find the whole energy spectra for these potentials, as in the case of the double-Morse potentials. 
Another possible application consists in taking the solutions for the GSWE as a guide to find new solutions for the (general) Heun equation, since the former equation is a confluent case of the latter. This would complement the procedure presented in the preceding sections, because eventually it would add the connection: general Heun equation $\rightarrow$ GSWE into the diagram (10). In fact, this link is required by the study of Teukolsky equations for gravitational backgrounds of black holes, as we have mentioned in the first section. Although the Heun equation is not subject of the present paper, in Appendix B we have introduced some preliminary results which indicate how the above goal could be attained.

\section{Acknowledgments}

I am indebted to Dr. Léa Jaccoud El-Jaick for her collaboration concerning results of section VI.B and Appendix B. I also thank Dr. Herman J. M. Cuesta for his reading of the manuscript and fruitful criticisms.

\section{Appendix A: Solutions in series of Bessel functions}

To find directly the solution $U_{1 \nu}^{\infty}(z)$ for the Whittaker-Ince limit of the generalized spheroidal wave equation,

$$
z\left(z-z_{0}\right) \frac{d^{2} U}{d z^{2}}+\left(B_{1}+B_{2} z\right) \frac{d U}{d z}+\left[B_{3}+q\left(z-z_{0}\right)\right] U=0,(q \neq 0),
$$

we perform the substitutions

$$
t=2 i \sqrt{q z}, \quad U_{1 \nu}^{\infty}(z)=t^{1-B_{2}} Y(t)
$$

to obtain

$$
\begin{aligned}
& t^{2} \frac{d^{2} Y}{d t^{2}}+t \frac{d Y}{d t}-t^{2} Y=-4 q z_{0} \frac{d^{2} Y}{d t^{2}}-\frac{4 q\left(z_{0}-2 B_{1}-2 B_{2} z_{0}\right)}{t} \frac{d Y}{d t} \\
& +\left[4 q\left(1-B_{2}\right) \frac{2 B_{1}+B_{2} z_{0}+z_{0}}{t^{2}}+\left(1-B_{2}\right)^{2}+4 q z_{0}-4 B_{3}\right] Y
\end{aligned}
$$

Next, by expanding $Y(t)$ in series of modified Bessel functions of the second kind $K_{\lambda}(t)$ according to

$$
Y(t)=\sum_{n=0}^{\infty} d_{n} t^{-n} K_{\lambda}(t), \text { where } \lambda=n+B_{2}-1
$$

we find

$$
\frac{d Y}{d t}=\sum_{n=0}^{\infty} d_{n} t^{-n}\left[\frac{d K_{\lambda}}{d t}-\frac{n}{t} K_{\lambda}\right], \frac{d^{2} Y}{d t^{2}}=\sum_{n=0}^{\infty} d_{n} t^{-n}\left[\frac{d^{2} K_{\lambda}}{d t^{2}}-\frac{2 n}{t} \frac{d K_{\lambda}}{d t}+\frac{n(n+1)}{t^{2}} K_{\lambda}\right] .
$$

Inserting (A3) and (A4) into the differential equation for $Y(t)$ and using the modified Bessel equation,

$$
t^{2} \frac{d^{2} K_{\lambda}}{d t^{2}}+t \frac{d K_{\lambda}}{d t}-t^{2} K_{\lambda}=\lambda^{2} K_{\lambda}
$$


it results

$$
\begin{aligned}
& \sum_{n=0}^{\infty} d_{n} t^{-n}\left[\lambda^{2}+n^{2}+4 B_{3}-4 q z_{0}-\left(1-B_{2}\right)^{2}\right] K_{\lambda}= \\
& \sum_{n=0}^{\infty} d_{n} t^{-n}\left[-4 q z_{0} \frac{d^{2} K_{\lambda}}{d t^{2}}+2 n t \frac{d K_{\lambda}}{d t}+4 q\left(2 n z_{0}-z_{0}+2 z_{0} B_{2}+2 B_{1}\right) \frac{1}{t} \frac{d K_{\lambda}}{d t}\right] \\
& -4 q \sum_{n=0}^{\infty} d_{n} t^{-n-2}\left[n\left(z_{0} n+2 z_{0}+2 B_{1}\right)-\left(1-B_{2}\right)\left(z_{0} B_{2}+2 B_{1}+z_{0}\right)\right] K_{\lambda} .
\end{aligned}
$$

From the difference-differential relations for $K_{\lambda}$ [39], we find that

$$
\begin{aligned}
\frac{d^{2} K_{\lambda}}{d t^{2}} & =K_{\lambda}+\lambda(\lambda-1) t^{-2} K_{\lambda}+t^{-1} K_{\lambda+1} \\
t \frac{d K_{\lambda}}{d t} & =-\lambda K_{\lambda}-t K_{\lambda-1} \\
\frac{1}{t} \frac{d K_{\lambda}}{d t} & =\lambda t^{-2} K_{\lambda}-t^{-1} K_{\lambda+1} .
\end{aligned}
$$

Thence, taking into account that $\lambda=n+B_{2}-1$, Eq. (A5) simplifies to

$$
\begin{aligned}
& \sum_{n=1}^{\infty} \frac{n}{2} d_{n} t^{-n+1} K_{n+B_{2}-2}+\sum_{n=0}^{\infty}\left[n\left(n+B_{2}-1\right)+B_{3}\right] d_{n} t^{-n} K_{n+B_{2}-1}+ \\
& \sum_{n=0}^{\infty}\left[2 z_{0} q\left(n+B_{2}+\frac{B_{1}}{z_{0}}\right)\right] d_{n} t^{-n-1} K_{n+B_{2}}=0
\end{aligned}
$$

since the coefficient of $t^{-n-2} K_{\lambda}$ vanishes. By the replacements $n \rightarrow m+1$ and $n \rightarrow m-1$ in the first and third terms, respectively, this equation becomes

$$
\begin{aligned}
& {\left[\frac{1}{2} d_{1}+B_{3} d_{0}\right] K_{B_{2}-1}+\sum_{n=1}^{\infty}\left\{\frac{1}{2}(n+1) d_{n+1}+\left[n\left(n+B_{2}-1\right)+B_{3}\right] d_{n}+\right.} \\
& \left.2 z_{0} q\left(n+B_{2}+\frac{B_{1}}{z_{0}}-1\right) d_{n-1}\right\} t^{-n} K_{n+B_{2}-1}=0 .
\end{aligned}
$$

Requiring that the coefficient of each independent term of this equation to vanish, we get the relations $\left(d_{-1}=0\right)$

$$
\frac{1}{2}(n+1) d_{n+1}+\left[n\left(n+B_{2}-1\right)+B_{3}\right] d_{n}+2 z_{0} q\left(n+B_{2}+\frac{B_{1}}{z_{0}}-1\right) d_{n-1}=0, n \geq 0 .
$$

Then, from Eqs. (A2) and (A3) we have

$$
U_{1 \nu}^{\infty}(z(t))=t^{1-B_{2}} \sum_{n=0}^{\infty} d_{n} t^{-n} K_{n+B_{2}-1,}(t) \propto z^{\left(1-B_{2}\right) / 2} \sum_{n=0}^{\infty} 2^{-n} d_{n}(i \sqrt{q z})^{-n} K_{n+B_{2}-1,}(2 i \sqrt{q z}) .
$$

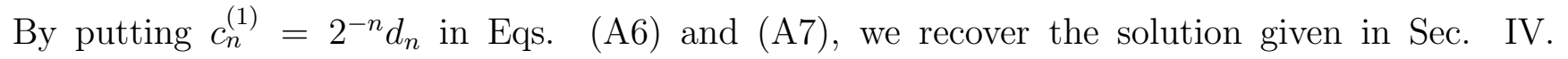
The derivation of the expansion in series of $J_{\lambda}(2 \sqrt{q z})$ is similar to the previous one. Thus, the Whittaker-Ince limits found in Sec. IV in fact satisfy the differential equation (A1). 


\section{Appendix B: General and confluent Heun equations}

The relationship between solutions of general Heun equation and GSWE (confluent Heun) is interesting by itself and becomes, in the context of relativistic astrophysics, important in the study of the Teukolsky equations of the gravitational backgrounds of black holes. In effect, for the Kerr metric these equations turn out to be GSWEs which afford DCHEs in the extreme upper limit of the rotation parameter [1]. On the other hand, the Teukolsky equations for the Kerr-de Sitter metric are (general) Heun equations which, in turn, reduce to GSWEs when the cosmological constant $\Lambda$ vanishes [11, 12]. Therefore, in such problems we have the connection: Heun equation $\rightarrow$ GSWE $\rightarrow$ DCHE, and this requires a generalization of the diagram (10).

In the following we exhibit two Erdélyi's solutions for the Heun equation [47] which lead to the expansions in hypergeometric functions given Sec. VI for the GSWE. From these Erdélyi solutions we explain how to obtain two additional solutions which yield the expansions in series of confluent hypergeometric functions for the GSWE as well.

To begin with, let there be the Heun equation [15, 48, 49]

$$
\frac{d^{2} H}{d x^{2}}+\left(\frac{\gamma}{x}+\frac{\delta}{x-1}+\frac{\epsilon}{x-a}\right) \frac{d H}{d x}+\frac{\alpha \beta x-q}{x(x-1)(x-a)} H=0, \quad \epsilon=\alpha+\beta+1-\gamma-\delta
$$

where $a \in \mathbb{C} \backslash\{0,1\}$ and $x=0,1, a, \infty$ are regular singular points with indicial exponents given by $\{0,1-\gamma\},\{0,1-\delta\},\{0,1-\epsilon\}$ and $\{\alpha, \beta\}$, respectively. For $a=0$ and $a=1$ the equation can be reduced to the hypergeometric equation by changes of variables; also for $\epsilon=0$ and $q=a \alpha \beta$ it degenerates to the hypergeometric equation. There is no possibility of confusing the auxiliary parameter $q$ with the parameter $q$ of the Whittaker-Ince limits given in Eq. (6) neither the singularity parameter $a$ with the constant $a$ of the Mathieu equation (12) because the $q$ and $a$ of the Heun equation disappear in the confluent limit of the equation (see Eq. (B6 $)$ ).

As in Ref. [12, we denote by $H_{1 \nu}^{\{0,1\}}(x)$ and $\hat{H}_{1 \nu}^{\{a, \infty\}}(x)$ the two Erdélyi solutions in series of hypergeometric functions, where the superscripts mean that the first solution converges in a domain containing the singular points 0 and 1 , and the second, in a domain containing $a$ and $\infty$. These solutions are [47] (once more $\nu$ is different from integer or half-integer)

$$
\begin{aligned}
& H_{1 \nu}^{\{0,1\}}(x)=\sum_{n=-\infty}^{\infty} b_{n}^{(1)} F\left(-n-\nu-1+\frac{\gamma+\delta}{2}, n+\nu+\frac{\gamma+\delta}{2} ; \gamma ; x\right) \\
& \hat{H}_{1 \nu}^{\{a, \infty\}}(x)=\sum_{n=-\infty}^{\infty} \hat{b}_{n}^{(1)} x^{-n-\nu-(\gamma+\delta) / 2} \widetilde{F}\left(n+\nu+1+\frac{\delta-\gamma}{2}, n+\nu+\frac{\gamma+\delta}{2} ; 2 n+2 \nu+2 ; \frac{1}{x}\right),
\end{aligned}
$$

where

$$
\hat{b}_{n}^{(1)}=(-1)^{n} \Gamma\left(n+\nu+2-\frac{\gamma+\delta}{2}\right) \Gamma\left(n+\nu+1+\frac{\delta-\gamma}{2}\right) b_{n}^{(1)} .
$$


In the recurrence relations (91a) for $b_{n}^{(1)}$ the coefficients are

$$
\begin{aligned}
\alpha_{n}^{(1)}= & -\frac{\left(n+\nu+2-\frac{\gamma+\delta}{2}\right)\left(n+\nu+1-\alpha+\frac{\gamma+\delta}{2}\right)\left(n+\nu+1-\beta+\frac{\gamma+\delta}{2}\right)\left(n+\nu+1-\frac{\gamma-\delta}{2}\right)}{4(n+\nu+1)\left(n+\nu+\frac{3}{2}\right)}, \\
\beta_{n}^{(1)}= & \left(\frac{1}{2}-a\right)(n+\nu)(n+\nu+1)+\frac{1}{4}[2 \alpha \beta+(\alpha+\beta)(\gamma-\delta)+(\gamma+\delta)(1-\gamma)] \\
& -\frac{1}{4} a(\gamma+\delta)(2-\gamma-\delta)-q+\frac{(\gamma-\delta)(\gamma+\delta-2)(2 \alpha-\gamma-\delta)(2 \beta-\gamma-\delta)}{32(n+\nu)(n+\nu+1)}, \\
\gamma_{n}^{(1)}= & -\frac{\left(n+\nu+\alpha-\frac{\gamma+\delta}{2}\right)\left(n+\nu+\beta-\frac{\gamma+\delta}{2}\right)\left(n+\nu-1+\frac{\gamma+\delta}{2}\right)\left(n+\nu+\frac{\gamma-\delta}{2}\right)}{4\left(n+\nu-\frac{1}{2}\right)(n+\nu)} .
\end{aligned}
$$

In these solutions the parameter $\nu$ which appears in some other works, for example in Ref. [15], have been replaced by the expression on right-hand side of the relation

$$
\nu \rightarrow \nu+1-\frac{\gamma+\delta}{2}
$$

This redefinition is due to Suzuki, Takasugi and Umetsu [12. It is useful to get the limits for the GSWE, as well as to obtain new solutions from the preceding ones by means of transformations like that written in Eq. (B11), simply because it is not necessary to transform $\nu$ in order to get solutions having the same series coefficients.

The above solutions are valid only if $a \notin[0,1]$ [47]. Since this restriction comes from the analysis of the recurrence relations for the coefficients $b_{n}^{(1)}$ as $n \rightarrow \pm \infty$, it is transferred to solutions whose coefficients are proportional to $b_{n}^{(1)}$. The solution $H_{1 \nu}^{\{0,1\}}(x)$ converges in the interior of an ellipse having foci at 0 and 1 and passing through the point $a$, provided that the characteristic equation is satisfied; the solution $\hat{H}_{1 \nu}^{\{a, \infty\}}(x)$ converges in the entire complex plane excepting the line joining the points 0 to 1 [47]. Therefore, we are dealing with a pair of solutions convergent over different domains.

To derive the GSWE as a confluence of Heun's equation (B1), first we rewrite the latter as

$x(x-1)\left(\frac{x}{a}-1\right) \frac{d^{2} H}{d x^{2}}+\left[\gamma(x-1)\left(\frac{x}{a}-1\right)+\delta x\left(\frac{x}{a}-1\right)+\frac{\epsilon}{a} x(x-1)\right] \frac{d H}{d x}+\left[\alpha \frac{\beta}{a} x-\frac{q}{a}\right] H=0$.

Then, by letting that

$$
a, \beta, q \rightarrow \infty \text { such that } \frac{\beta}{a} \rightarrow \frac{\epsilon}{a} \rightarrow-\rho, \frac{q}{a} \rightarrow-\sigma, H(x) \rightarrow h(x),
$$

$\rho$ and $\sigma$ being constants, we find the GSWE [15]

$$
x(x-1) \frac{d^{2} h}{d x^{2}}+[-\gamma+(\gamma+\delta) x+\rho x(x-1)] \frac{d h}{d x}+[\alpha \rho x-\sigma] h=0,
$$

which, however, is not in the form suitable to get the DCHE by means of the Leaver limit. Despite this, from the previous solutions, we see that $H(x)$ and its limit $h(x)$ have the same functional form. To obtain the recurrence relations for the series coefficients, first we divide the coefficients (B4) by $a$ and then take the limits of $\alpha_{n}^{(1)} / a, \beta_{n}^{(1)} / a$ and $\gamma_{n}^{(1)} / a$ when $a \rightarrow \infty$, by using the relations (B6). To permit the Leaver limit, the second step is given by the substitutions

$$
x=\frac{z_{0}-z}{z_{0}}, \quad h(x)=e^{\rho z /\left(2 z_{0}\right)} U(z)
$$


which convert Eq. (B7) into

$z\left(z-z_{0}\right) \frac{d^{2} U}{d z^{2}}+\left[-\delta z_{0}+(\gamma+\delta) z\right] \frac{d U}{d z}+\left[\frac{\gamma \rho}{2}-\sigma-\left(\alpha-\frac{\gamma+\delta}{2}\right) \frac{\rho\left(z-z_{0}\right)}{z_{0}}-\frac{\rho^{2} z\left(z-z_{0}\right)}{4 z_{0}^{2}}\right] U=0$.

Then, comparing this with the GSWE (1), we find that

$$
\alpha=i \eta+\frac{B_{2}}{2}, \delta=-\frac{B_{1}}{z_{0}}, \quad \gamma=B_{2}+\frac{B_{1}}{z_{0}}, \quad \rho=-2 i \omega z_{0}, \quad \sigma=-i \omega z_{0}\left(B_{2}+\frac{B_{1}}{z_{0}}\right)-B_{3} .
$$

Therefore, thanks to Eq. (B8), the solutions $U(z)$ for the GSWE (1) are obtained by writing

$$
U\left(B_{1}, B_{2}, B_{3} ; z_{0}, \omega, \eta ; z\right)=e^{i \omega z} h\left(\alpha, \gamma, \delta, \rho, \sigma ; x=\frac{z_{0}-z}{z}\right)
$$

and by inserting the parameters (Bי $)$ into the right-hand side and also into the recurrence relations obtained in the first step.

By this procedure, the solutions $H_{1 \nu}^{\{0,1\}}(x)$ and $H_{1 \nu}^{\{a, \infty\}}(x)$ for the Heun equation give, respectively, the solutions $U_{1 \nu}^{0}(z)$ and $\hat{U}_{1 \nu}^{\infty}(z)$ for the GSWE, written in Sec. VI . To obtain solutions which lead to the expansions $U_{1 \nu}^{\infty}(z)$ and $\widetilde{U}_{1 \nu}^{\infty}(z)$ for the GSWE, we achieve an appropriate substitution of the independent variable, followed by a transformation of the dependent variable, such that the Heun equation is transformed into another version of itself (with a different set of parameters). More precisely, from the transformation theory for the Heun equation [49], if $H(a, q ; \alpha, \beta, \gamma, \delta ; x)$ is a solution of the Heun equation, then another solution is obtained by means of (transformation number 105 of Maier's table [49])

$$
\begin{aligned}
& H(a, q ; \alpha, \beta, \gamma, \delta ; x) \rightarrow \\
& (x-1)^{-\alpha} H\left(a, q+\alpha(\alpha-\gamma-\delta+1) a ; \alpha, \alpha-\delta+1, \epsilon, \alpha-\beta+1 ; \frac{x-a}{x-1}\right)
\end{aligned}
$$

Then, by identifying $H(a, q ; \alpha, \beta, \gamma, \delta ; x)$ with $H_{1}^{\{0,1\}}(x)$ and $\hat{H}_{1}^{\{a, \infty\}}(x)$ we find that

$$
\begin{gathered}
H_{1 \nu}^{\{0,1\}}(x) \rightarrow(x-1)^{-\alpha} \sum_{n=-\infty}^{\infty} b_{n}^{(1)} F\left(-n-\nu+\alpha-\frac{\gamma+\delta}{2}, n+\nu+1+\alpha-\frac{\gamma+\delta}{2} ; \epsilon ; \frac{x-a}{x-1}\right), \\
\hat{H}_{1 \nu}^{\{a, \infty\}}(x) \rightarrow(x-1)^{-\alpha} \sum_{n=-\infty}^{\infty} c_{n}^{(1)}\left[\frac{1-\frac{x}{a}}{x-1}\right]^{-n-\nu-\alpha-1+\frac{\gamma+\delta}{2}} \times \\
\widetilde{F}\left(n+\nu+1+\alpha-\frac{\delta+\gamma}{2}, n+\nu+1-\beta-\frac{\gamma+\delta}{2} ; 2 n+2 \nu+2 ; \frac{x-1}{x-a}\right),
\end{gathered}
$$

where

$$
c_{n}^{(1)}=(a)^{n} \Gamma\left(n+\nu+1-\alpha-\frac{\gamma+\delta}{2}\right) \Gamma\left(n+\nu+1-\beta+\frac{\delta+\gamma}{2}\right) b_{n}^{(1)} .
$$

Note that $\nu$ has not been transformed and that the coefficients $b_{n}^{(1)}$ are the same which appear in the original solutions.

Following the procedure outline above for $a \rightarrow 0$ and using the limits (98) for the hypergeometric functions $F(a, b ; c ; y)$, we can show that these give the solutions $U_{1 \nu}^{\infty}(z)$ and $\widetilde{U}_{1 \nu}^{\infty}(z)$ in series 
of confluent hypergeometric functions for the GSWE. In the first solution, before taking the limit of the hypergeometric functions we make the approximations

$$
c=\epsilon=-\rho a, \quad \frac{x-a}{x-1}=1+\frac{1-a}{x-1} \sim 1-\frac{c}{\rho(1-x)} .
$$

In the second solution, first we must take

$$
b \sim-\beta=\rho a, \quad \frac{x-1}{x-a} \sim \frac{\rho(1-x)}{b}
$$

and, in addition, we must find the recurrence relations for the coefficients $c_{n}^{(1)}$ defined by Eq. (B13). However, to establish precisely this first set of solutions, it is necessary to study the convergence of the two last solutions and, then, apply the so-called elementary power transformations of the dependent variable [15] in order to generate new sets of solutions. We have also to find solutions valid for $a \in(0,1)$.

Finally we mention that the following series solution for the Heun equation [15]

$$
H^{\{0,1\}}(x)=\sum_{n=0}^{\infty} d_{n} x^{n}, \quad|a|>1,
$$

where the coefficients satisfy the relations

$$
\begin{aligned}
& a(n+1)(n+\gamma) d_{n+1}-[\operatorname{an}(n+\gamma+\delta-1)+n(n+\alpha+\beta-\delta)+q] d_{n} \\
& +(n+\alpha-1)(n+\beta-1) d_{n-1}=0,
\end{aligned}
$$

converges for $|x|<|a|$ and gives, by the above procedure, the solution $U_{1}^{0}(z)$ of section II for the GSWE. By analogy with the previous case, we may expect that the generalization of the solutions $U_{1}^{\infty}(z)$ and $U_{1}(z)$ (in series of confluent hypergeometric functions) are given by series of hypergeometric functions .

\section{References}

[1] E. W. Leaver, J. Math. Phys. 27, 1238 (1986).

[2] P. Humbert, Fonctions de Lamé et Fonctions de Mathieu, Mémorial des Sciences Mathématiques, X (Gauthier-Villards, Paris, 1926).

[3] E. L. Ince, Proc. Lond. Math. Soc. 23, 56 (1923).

[4] B. D. B. Figueiredo, J. Math. Phys. 46, 113503 (2005).

[5] A. Decarreau, P. Maroni and A. Robert, Ann. Soc. Sci. Bruxelles, Ser. 1 T92, 151 (1978) .

[6] O. B. Zaslavskii and V. V. Ulyanov, Sov. Phys. JETP 60, 991 (1984).

[7] V. V. Ulyanov and O. B. Zaslavskii, Phys. Rep. 261, 179 (1992).

[8] A. G. Ushveridze, Sov. J. Part. Nucl. 20, 504 (1989). 
[9] A. G. Ushveridze, Quasi-Exactly Solvable Models in Quantum Mechanics (IOP, Bristol, 1994).

[10] B. D. B. Figueiredo, J. Phys. A: Math. Gen. 35, 2877, (2002); J. Phys. A: Math. Gen. 35, 4799 (2002) (corrigendum).

[11] H. Suzuki. E. Takasugi and H. Hiroshi, Prog. Theor. Phys. 100, 491 (1998).

[12] H. Suzuki, E. Takasugi and H. Umetsu, Prog. Theor. Phys. 102, 253 (1999).

[13] F. M. J. Olver, Asymptotics and Special Functions (Academic Press, New York, 1974).

[14] A. Decarreau, M. C. Dumont-Lepage, P. Maroni, A. Robert and A. Ronveaux, Ann. Soc. Sci. Bruxelles, Ser. 1 T92, 53 (1978).

[15] Heun's Differential Equations, edited by A. Ronveaux (Oxford University Press, 1995).

[16] A. H. Wilson, Proc. Roy. Soc. London A118, 617 (1928).

[17] D. Schmidt and G. Wolf, Double Confluent Heun Equation, Part C of [15].

[18] S. Mignemi, J. Math. Phys. 32, 3047 (1991).

[19] A. Malmendier, J. Math. Phys. 44, 4308 (2003).

[20] T. Eguchi and A. J. Hanson, Phys. Lett. 74B, 249 (1978) .

[21] F. M. Arscott, Proc. Roy. Soc. Edinburg A67, 265 (1967) .

[22] E. W. McLachlan, Theory and Application of Mathieu Functions (Dover, New York, 1964).

[23] E. L. Ince, Proc. Lond. Math. Soc. 25, 53 (1926) .

[24] W. G. Barber and H. R. Hassé, Proc. Camb. Phil. Soc. 25, 564 (1935 ).

[25] V. S. Otchik, in Quantum Systems: New Trends and Methods, edited by A. O. Barut, I. D. Feranchuk, Ya. M. Shnir and L. T. Tomil'chik (World Scientific, 1995).

[26] S. Mano, H. Suzuki and E. Takasugi, Prog. Theor. Phys. 95, 1079 (1996).

[27] S. Mano, H. Suzuki and E. Takasugi, Prog. Theor. Phys. 96, 549 (1996).

[28] S. Mano and E. Takasugi, Prog. Theor. Phys. 97, 213 (1997).

[29] F. Lindemann, Math. Ann. 22, 117 (1883).

[30] E. T. Whittaker and G. N. Watson, A Course of Modern Analysis (Cambridge University Press, 1945).

[31] E. Fisher, Phil.Mag. 24, 245 (1937).

[32] D. Bini, C. Cherubini, R. T. Jantzen, B. Mashhoon, Phys. Rev. D67, 084013 (2003).

[33] W. Gautschi, SIAM Rev. 9, 24 (1967).

[34] F. M. Arscott, Periodic Differential Equations (Macmillan Company, New York, 1964). 
[35] A. Erdélyi, W. Magnus, F. Oberhettinger and F. G. Tricomi (Bateman Manuscript Project) Higher Transcendental Functions, vol. 1 (McGraw-Hill, New York, 1953).

[36] Handbook of Mathematical Functions, edited by M. Abramowitz and I. A. Stegun (Dover, New York, 1965 ).

[37] I. S. Gradshteyn and I. M. Ryzhik, Table of Integrals, Series and Products (Academic Press, New York, 1994).

[38] A. Lemieux and A. K. Bose, Ann. Inst. Henri Poincaré 10, 259 (1969) .

[39] Y. L. Luke, Integrals of Bessel functions (McGraw-Hill, New York, 1962).

[40] E. Schrödinger, Commentationes Pontificiae Academiae Scientiarum, 2, 321 (1938).

[41] E. Schrödinger, Proceedings of the Royal Irish Academy A46, 25 (1940).

[42] N. D. Birrell and P. C. W. Davies, Quantum Fields in Curved Space (Cambridge University Press, 1982).

[43] C. J. Kleinman, Y. Hahn and L. Spruch, Phys. Rev. 165, 53 (1968).

[44] W. Bühring, J. Math. Phys. 15, 1451 (1974).

[45] W. Bühring, in Centennial Workshop on Heun's Equation, edited by A. Seeger and W. Lay (Max-Plank-Institut für Metallforchung, Institut für Physik, Stuttgart, 1990).

[46] E. D. Fackerell and R. G. Crossman, J. Math. Phys. 18, 1849 (1977).

[47] A. Erdélyi, Q. J. Math. 15, 62 (1944).

[48] K. Heun, Math. Ann. 33, 161 (1899).

[49] R. S. Maier, e-print math.CA/0408317 v2 (2006). 